\title{
Long-term visit-to-visit glycemic variability as predictor of micro- and macrovascular complications in patients with type 2 diabetes: The Rio de Janeiro Type 2 Diabetes Cohort Study
}

\author{
C. R. L. Cardoso ${ }^{1}$, N. C. Leite ${ }^{1}$, C. B. M. Moram² and G. F. Salles ${ }^{1 *}$
}

\begin{abstract}
Background: Long-term visit-to-visit glycemic variability is an additional measure of glycemic control. We aimed to evaluate the prognostic value of several measures of glycemic variability for the occurrence of micro- and macrovascular complications, and all-cause mortality in patients with type 2 diabetes.

Methods: 654 individuals were followed-up over a median of 9.3 years. Glycemic variability (SDs and coefficients of variation of $\mathrm{HbA}_{1 \mathrm{c}}$ and fasting glycaemia) was measured during the first 12- and 24-months. Multivariate Cox analysis, adjusted for risk factors and mean $\mathrm{HbA}_{1 c}$ and fasting glycaemia levels, examined the associations between glycemic variability and the occurrence of microvascular (retinopathy, microalbuminuria, renal function deterioration, peripheral neuropathy) and macrovascular complications [total cardiovascular events (CVE), major adverse CVEs (MACE) and cardiovascular mortality], and of all-cause mortality.

Results: During follow-up, 128 patients had a CVE (96 MACE), and 158 patients died (67 from cardiovascular diseases); 152 newly-developed or worsened diabetic retinopathy, 183 achieved the renal composite outcome (89 newly developed microalbuminuria and 91 deteriorated renal function), and 96 newly-developed or worsened peripheral neuropathy. Glycemic variability, particularly the 24-month parameters either estimated by $\mathrm{HbA}_{1 c}$ or by fasting glycemia, predicted all endpoints, except for retinopathy and peripheral neuropathy development/progression, and was a better predictor than mean $\mathrm{HbA}_{1 c}$. Glycemic variability predicted retinopathy development/progression in patients with good glycemic control $\left(\mathrm{HbA}_{1 \mathrm{c}} \leq 7.5 \%, 58 \mathrm{mmol} / \mathrm{mol}\right)$ and predicted new-incident peripheral neuropathy.

Conclusions: Long-term visit-to-visit glycemic variability is an additional and frequently a better glycemic parameter than mean $\mathrm{HbA}_{1 \mathrm{c}}$ levels for assessing the risk of future development of micro- and macrovascular complications in patients with type 2 diabetes.
\end{abstract}

Keywords: Glycemic variability, Macrovascular complications, Microvascular complications, Mortality, Type 2 diabetes

\footnotetext{
*Correspondence: gilsalles@hucff.ufrj.br

${ }^{1}$ Department of Internal Medicine, University Hospital Clementino Fraga Filho, School of Medicine, Universidade Federal do Rio de Janeiro, Rua

Croton, 72, Jacarepagua, Rio de Janeiro, RJ CEP: 22750-240, Brazil

Full list of author information is available at the end of the article
} 


\section{Background}

Type 2 diabetes is an important public health problem worldwide, not only by its increasing prevalence, but also because its associated micro- and macrovascular complications severely impact on individuals' quality of life and pose a great burden on healthcare systems $[1,2]$. Current diabetes treatment aims to reducing chronic complications development and progression, mainly by controlling hyperglycemia, high blood pressure (BP) levels and dyslipidemia; and glycemic control is traditionally monitored by serial mean glycated hemoglobin $\left(\mathrm{HbA}_{1 \mathrm{c}}\right)$ levels [3]. This rationale comes from several trials and observational studies showing that lowering $\mathrm{HbA}_{1 \mathrm{c}}$ indeed reduces the risk of micro- and macrovascular complications [4-6]. However, particularly for macrovascular disease, there is no consensus that lowering HbA1c to levels below $8.5-8.0 \%(69-64 \mathrm{mmol} / \mathrm{mol})$ actually reduces such risk $[7,8]$. Moreover, even for microvascular complications there may be additional glycemic factors associated with increased risks, beyond mean HbA1c levels $[9,10]$. In this context, the concept of glycemic variability has recently emerged as another measure of glycemic control, which might constitute an additive, or even better predictor of diabetic complications than mean HbA1c levels $[11,12]$.

Glycemic variability is a general denomination to several measures of short-term and long-term fluctuations in glycemia. Short-term glycemic variability refers to within-day or between-days glycemia fluctuations, and is usually measured by continuous glucose monitoring mainly in individuals with type 1 diabetes [12]. Long-term glycemic variability refers to glycemic fluctuations over months to years and is generally measured by visit-to-visit variability in either $\mathrm{HbA}_{1 \mathrm{c}}$ or fasting glycemia (FG) in both type 1 and type 2 diabetes [12]. Several previous studies in patients with type 2 diabetes [13-41] showed that long-term glycemic variability may predict both microvascular (retinopathy [13-18], diabetic kidney disease [16, 18-26], and cardiovascular autonomic neuropathy [27]) and macrovascular complications [23, 28-36], as well as all-cause mortality [29, 32-34, 36-41]. However, there were conflicting results, particularly regarding diabetic retinopathy [14, 16-18] and cardiovascular disease incidence [33-36]. Moreover, except for a few reports [16, 18, 23, 29, 32-34, 36], most of the previous studies evaluated only a single diabetic complication, without a more comprehensive analysis of several adverse diabetic complications. Only a recent meta-analysis [12] provided such extensive examination. Finally, the value of glycemic variability as a predictor of future diabetic peripheral neuropathy development and progression, an important microvascular complication, as far as we know, has not been evaluated yet. In resume, the importance of long-term visit-to-visit glycemic variability measures as predictors of future complications in patients with type 2 diabetes still remains controversial $[42,43]$, and further studies are needed.

Therefore, the aim of this study was to evaluate the prognostic value of several measures of long-term glycemic variability for the occurrence of separated microvascular (retinopathy, microalbuminuria, renal function deterioration, and peripheral neuropathy) and macrovascular complications (total cardiovascular events, major cardiovascular events, cardiovascular mortality), and all-cause mortality in The Rio de Janeiro Type 2 Diabetes (RIO-T2D) Cohort Study, an on-going cohort of high cardiovascular risk individuals with type 2 diabetes with a median follow-up of nearly 10 years.

\section{Methods}

\section{Patients and baseline procedures}

This was a prospective study, nested within The Rio de Janeiro Type 2 Diabetes Cohort Study, with 654 patients with type 2 diabetes enrolled between August 2004 and December 2008 and re-evaluated annually until December 2016 in the diabetes outpatient clinic of our tertiarycare University Hospital. All participants gave written informed consent, and the local Ethics Committee had previously approved the study protocol. The characteristics of this cohort, the baseline procedures and the diagnostic definitions have been detailed elsewhere [44-47]. In brief, inclusion criteria were all adult type 2 diabetic individual up to 80 years old with either any microvascular (retinopathy, nephropathy or neuropathy) or macrovascular (coronary, cerebrovascular or peripheral artery disease) complication, or with at least two other modifiable cardiovascular risk factors. Exclusion criteria were morbid obesity (body mass index $\geq 40 \mathrm{~kg} / \mathrm{m}^{2}$ ), advanced renal failure (serum creatinine $>180 \mu \mathrm{mol} / \mathrm{l}$ or estimated glomerular filtration rate $<30 \mathrm{ml} / \mathrm{min} / 1.73 \mathrm{~m}^{2}$ ) or the presence of any serious concomitant disease limiting life expectancy. All were submitted to a standard baseline protocol that included a thorough clinical examination, a laboratory evaluation, and a 24-h ambulatory BP monitoring (ABPM). Diagnostic criteria for diabetic chronic complications were detailed previously [44-47]. In brief, coronary heart disease was diagnosed by clinical, electrocardiographic criteria, or by positive ischemic stress tests. Cerebrovascular disease was diagnosed by history and physical examination, and peripheral arterial disease by an ankle-brachial index $<0.9$. The diagnosis of nephropathy needed at least two albuminurias $\geq 30 \mathrm{mg} / 24 \mathrm{~h}$ or proteinurias $\geq 0.5 \mathrm{~g} / 24 \mathrm{~h}$ or confirmed reduction of glomerular filtration rate $(\mathrm{eGFR} \leq 60 \mathrm{ml} /$ $\min / 1.73 \mathrm{~m}^{2}$, estimated by the CKD-EPI equation, or serum creatinine $>130 \mu \mathrm{mol} / \mathrm{l})$. Peripheral neuropathy 
was determined by clinical examination (knee and ankle reflex activities, feet sensation with the Semmes-Weinstein monofilament, vibration with a $128-\mathrm{Hz}$ tuning fork, pinprick and temperature sensations) and neuropathic symptoms were assessed by a standard validated questionnaire [45]. Clinic blood pressure (BP) was measured three times using a digital oscillometric BP monitor (HEM-907XL, Omron Healthcare, Kyoto, Japan) with a suitable sized cuff on two occasions 2 weeks apart at study entry. The first measure of each visit was discarded and BP considered was the mean between the last two readings of each visit. Arterial hypertension was diagnosed if mean systolic (SBP) $\geq 140 \mathrm{mmHg}$ or diastolic $\mathrm{BP}(\mathrm{DBP}) \geq 90 \mathrm{mmHg}$ or if anti-hypertensive drugs had been prescribed. ABPM was recorded in the following month using Mobil-O-Graph, version 12 equipment (Dynamapa, Cardios LTDA., São Paulo, Brazil), and average 24-h SBP and DBP were registered [47]. Laboratory evaluation included fasting glycemia (FG), glycated hemoglobin $\left(\mathrm{HbA}_{1 \mathrm{c}}\right)$, serum creatinine and lipids. Albuminuria and proteinuria were evaluated in two non-consecutive sterile 24-h urine collections.

\section{Long-term glycemic variability measurements}

The patients had at least three annual $\mathrm{HbA}_{1 \mathrm{c}}$ and $\mathrm{FG}$ measurements during follow-up. Long-term visit-to-visit glycemic variability was estimated separately for $\mathrm{HbA}_{1 \mathrm{c}}$ and FG, and for the first 12 and 24-month periods, as the standard deviation (SD) of all measurements performed during these periods. To account for the possible influence of different number of measurements, the SD was divided by $\sqrt{[n} /(n-1)]$, as previously suggested [10]. The coefficient of variation (SD/mean) was also calculated for each glycemic variability parameter.

\section{Follow-up and outcomes assessment}

The patients were followed-up regularly at least 3-4 times a year until December 2016 under standardized treatment. The observation period for each patient was the number of months from the date of the first clinical examination to the date of the last clinical visit in 2016 or the date of the first endpoint, whichever came first. The primary endpoints were the occurrence of any macrovascular or microvascular outcomes. Macrovascular outcomes were total cardiovascular events (CVEs: fatal or non-fatal myocardial infarctions, sudden cardiac deaths, new-onset heart failure, death from progressive heart failure, any myocardial revascularization procedure, fatal or non-fatal strokes, any aortic or lower limb revascularization procedure, any amputation above the ankle, and deaths from aortic or peripheral arterial disease), major adverse cardiovascular events (MACE: non-fatal myocardial infarctions and strokes plus cardiovascular deaths), and all-cause and cardiovascular mortalities [44]. Mortality, as well as non-fatal cardiovascular events occurrence, was ascertained from medical records, death certificates and interviews with attending physicians and patient families, by a standard questionnaire reviewed by two independent observers. In case of disagreement, it was decided by consensus with a third independent consultant. Most of the in-hospital fatal or non-fatal events were attended at our own hospital. Microvascular outcomes were retinopathy development or worsening [46], renal outcomes [47] [new microalbuminuria development, new renal failure development (defined as doubling of serum creatinine or end-stage renal failure needing dialysis or death from renal failure), and a composite of them], and peripheral neuropathy development or worsening [45]. Retinopathy and renal outcomes were evaluated by annual examinations [46, 47], whereas peripheral neuropathy was evaluated on a second specific examination performed after a median of 6 years from the baseline examination [45].

\section{Statistical analyses}

Continuous data were described as means (SD) or as medians (interquartile range). For initial exploratory analyses, patients were categorized into tertiles of glycemic variability parameters and baseline characteristics compared by ANOVA, Kruskal-Wallis or $\chi^{2}$ tests, when appropriate. Kaplan-Meier curves of cumulative endpoints incidence during follow-up, compared by log-rank tests, were used for assessing different incidences of outcomes among tertile subgroups. For assessing the prognostic value of each glycemic variability parameter for each macrovascular and microvascular outcome, except for peripheral neuropathy, a time-to-event Cox analysis was undertaken with progressively increasing statistical adjustments for potential confounding. Model 1 was only adjusted for age, sex and number of $\mathrm{HbA}_{1 \mathrm{c}}$ or FG measurements, model 2 was further adjusted for other potential confounders (diabetes duration, body mass index (BMI), smoking status, physical inactivity, arterial hypertension, number of anti-hypertensive drugs in use, ambulatory 24-h SBP, presence of each micro- and macrovascular complications at baseline, serum mean HDL- and LDLcholesterol, and use of insulin, statins and aspirin), and model 3 was further adjusted for mean FG and $\mathrm{HbA}_{1 \mathrm{c}}$ levels during the same period of glycemic variability measurement. These results were presented as hazard ratios (HRs) with their 95\% confidence intervals (CIs); 
to allow comparisons among different glycemic variability parameters, their HRs were calculated for standardized increments of 1-SD. As glycemic variability was measured during the first 2 years of follow-up, patients who presented any of the endpoints during this period were excluded from the analysis of this specific outcome. For peripheral neuropathy analyses, a multiple logistic regression was used with the same progressively increasing statistical adjustments, except that height (instead of BMI) and the time interval between the baseline and second neuropathy evaluations were included as adjusting covariates. These results were reported as odds ratios (ORs) with their respective 95\% CIs, also estimated for increments of 1-SD in each glycemic variability parameter. The same analyses were performed for patients categorized into tertiles of each glycemic variability parameter, with HRs and ORs calculated for the highest tertile subgroup in relation to the lowest tertile reference subgroup, after adjustments for the same covariates. Interaction between mean $\mathrm{HbA}_{1 \mathrm{c}}$ and glycemic variability measures were tested for all endpoints and whenever there was evidence of interaction ( $\mathrm{p}<0.10$ for interaction term), stratified analyses for high $(>7.5 \%, 58 \mathrm{mmol} / \mathrm{mol})$ and low $(\leq 7.5 \%) \mathrm{HbA}_{1 \mathrm{c}}$ levels were performed. Statistics were performed with SPSS version 19.0 (SPSS Inc, Chicago, Il., USA), and a 2 -tailed probability value $<0.05$ was considered significant.

\section{Results}

\section{Baseline characteristics}

For macrovascular and mortality outcomes, 654 patients without any endpoint occurrence during the first 2 years of follow-up were evaluated. For microvascular outcomes, 615 patients were evaluated for renal, 533 for retinopathy and 471 for peripheral neuropathy outcomes. Patients had a median of $4 \mathrm{HbA}_{1 \mathrm{c}}$ (range 3-6) and 5 FG (range 3-7) measurements during the first 12 months of follow-up, and a median of $8 \mathrm{HbA}_{1 \mathrm{c}}$ (range 6-11) and $10 \mathrm{FG}$ (range 6-14) measurements during the first 24 months of follow-up. Median time interval between each visit-to-visit $\mathrm{HbA}_{1 \mathrm{c}}$ and FG measurements was 3 months. Table 1 outlines the baseline characteristics of all patients and of those divided according to tertiles of 24-month $\mathrm{HbA}_{1 \mathrm{c}}$ variability. Patients with higher $\mathrm{HbA}_{1 \mathrm{c}} \mathrm{SD}$ were younger, but with longer diabetes duration, and had higher prevalences of microvascular complications than those with lower $\mathrm{HbA}_{1 \mathrm{c}}$ variability. They also had higher $\mathrm{BP}$ levels, particularly at $\mathrm{ABPM}$, and poorer glycemic control, although using insulin more frequently, than those with lower $\mathrm{HbA}_{1 \mathrm{c}}$ variability. Table 2 shows the same baseline characteristics of patients divided according to 24-month FG variability. In general, they follow the same patterns of $\mathrm{HbA}_{1 \mathrm{c}}$ variability, except that patients with higher FG-SD also had greater prevalences of macrovascular complications than those with lower FG variability.

\section{Endpoints occurrence during follow-up}

Over a median follow-up of 9.3 years (IQR 5.210.8 years), 128 patients had a CVE (96 MACE), and 158 patients died (67 from cardiovascular diseases); 152 newly-developed or worsened diabetic retinopathy, 183 achieved the renal composite outcome (89 newly developed microalbuminuria and 91 deteriorated renal function), and 96 newly-developed or worsened peripheral neuropathy. Tables 1 and 2 show that patients with higher long-term glycemic variability had a significantly higher incidence of all endpoints, except of all-cause mortality and new microalbuminuria development for $\mathrm{HbA}_{1 \mathrm{c}}$ variability and of new peripheral neuropathy development for FG variability. Kaplan-Meier curves of cumulative incidence of endpoints (Figs. 1, 2) shows that for most of the endpoints the increased incidence was mainly observed in the highest tertile variability subgroup in relation to the middle and lowest tertile subgroups, except for retinopathy (for $\mathrm{HbA}_{1 \mathrm{c}}$ variability) and renal outcomes (for FG variability), where those patients in the middle tertile subgroup already had an increased incidence of these endpoints.

\section{Risks associated with increased long-term glycemic variability}

Table 3 (for macrovascular and mortality outcomes) and Table 4 (for microvascular outcomes) present the risks associated with a 1-SD increment in each 12- and 24-month glycemic variability parameter after increasing levels of confounding variables adjustments. As a whole, 24-month glycemic variability parameters were better risk predictors than 12-month parameters, and variabilities estimated by SDs and by CVs were roughly equivalent. For cardiovascular endpoints, particularly for MACE, 24-month glycemic variability, either estimated by $\mathrm{HbA}_{1 \mathrm{c}}$ or by $\mathrm{FG}$, were independent predictors 
Table 1 Characteristics of all diabetic patients and divided into tertiles of 24-month $\mathrm{HbA}_{1 \mathrm{c}}$ variability

\begin{tabular}{|c|c|c|c|c|c|}
\hline Characteristics & $\begin{array}{l}\text { All patients } \\
(n=654)\end{array}$ & $\begin{array}{l}\text { 1st-tertile } \\
\mathrm{HbA} A_{1 c}-\mathrm{SD} \\
\leq 0.45 \% \\
(\mathrm{n}=218)\end{array}$ & $\begin{array}{l}\text { 2nd-tertile } \\
\mathrm{HbA}_{1 \mathrm{c}}-\mathrm{SD} \\
0.46-0.85 \% \\
(\mathrm{n}=218)\end{array}$ & $\begin{array}{l}\text { 3rd-tertile } \\
\mathrm{HbA}_{1 c} \text {-SD } \\
\geq 0.86 \% \\
(\mathrm{n}=218)\end{array}$ & p value \\
\hline Age (years) & $60.1(9.6)$ & $61.2(9.4)$ & $59.9(9.9)$ & $58.7(9.4)$ & 0.007 \\
\hline Male sex (\%) & 38.1 & 42.2 & 37.6 & 34.4 & 0.26 \\
\hline $\mathrm{BMI}\left(\mathrm{kg} / \mathrm{m}^{2}\right)$ & $29.7(4.8)$ & $29.9(4.6)$ & $29.7(5.0)$ & $29.6(5.0)$ & 0.84 \\
\hline Smoking, current/past (\%) & 45.1 & 48.2 & 40.8 & 46.3 & 0.29 \\
\hline Physical activity (\%) & 22.4 & 23.4 & 23.5 & 20.2 & 0.64 \\
\hline Diabetes duration (years) & $8.0(3.0-15.0)$ & $5.0(1.4-12.3)$ & $10.0(4.0-17.5)$ & $9.0(5.0-15.0)$ & $<0.001$ \\
\hline \multicolumn{6}{|l|}{ Chronic diabetic complications (\%) } \\
\hline Cerebrovascular disease & 9.0 & 11.5 & 5.5 & 10.1 & 0.075 \\
\hline Coronary artery disease & 15.6 & 14.7 & 15.1 & 17.0 & 0.83 \\
\hline Peripheral artery disease & 17.0 & 15.7 & 17.1 & 18.3 & 0.76 \\
\hline Retinopathy & 32.7 & 27.1 & 34.1 & 36.7 & 0.087 \\
\hline Nephropathy & 31.0 & 22.0 & 27.3 & 43.6 & $<0.001$ \\
\hline Peripheral neuropathy & 29.0 & 26.5 & 31.2 & 29.2 & 0.56 \\
\hline Cardiovascular autonomic neuropathy & 18.1 & 14.9 & 17.4 & 21.8 & 0.24 \\
\hline \multicolumn{6}{|l|}{ Diabetes treatment (\%) } \\
\hline Metformin & 87.9 & 89.4 & 86.2 & 88.1 & 0.61 \\
\hline Sulfonylureas & 43.3 & 44.5 & 43.6 & 41.7 & 0.86 \\
\hline Insulin & 48.9 & 29.8 & 51.8 & 65.1 & $<0.001$ \\
\hline Aspirin & 90.9 & 91.2 & 87.6 & 94.0 & 0.068 \\
\hline Dyslipidemia (\%) & 87.3 & 87.2 & 86.7 & 88.1 & 0.93 \\
\hline Statins use (\%) & 77.5 & 79.7 & 75.6 & 77.1 & 0.57 \\
\hline Arterial hypertension (\%) & 86.5 & 84.4 & 88.1 & 87.2 & 0.51 \\
\hline Number of anti-hypertensive drugs & $3(1-4)$ & $3(1-4)$ & $3(1-4)$ & $3(1-4)$ & 0.97 \\
\hline ACE inhibitors/AR blockers (\%) & 83.0 & 82.0 & 82.0 & 85.0 & 0.65 \\
\hline Diuretics (\%) & 67.7 & 68.4 & 66.5 & 68.1 & 0.92 \\
\hline Calcium channel blockers (\%) & 31.7 & 35.4 & 33.0 & 26.6 & 0.14 \\
\hline Beta-blockers (\%) & 50.1 & 47.6 & 51.0 & 51.7 & 0.68 \\
\hline \multicolumn{6}{|l|}{ Blood pressures (mmHg) } \\
\hline Clinic SBP & $147(25)$ & $147(25)$ & $145(25)$ & $149(24)$ & 0.12 \\
\hline Clinic DBP & $84(13)$ & $83(13)$ & $84(13)$ & $86(14)$ & 0.072 \\
\hline Ambulatory 24 h SBP & $128(15)$ & $126(15)$ & $128(15)$ & $131(16)$ & 0.002 \\
\hline Ambulatory 24 h DBP & $74(10)$ & $72(9)$ & $74(10)$ & $75(11)$ & 0.009 \\
\hline \multicolumn{6}{|l|}{ Laboratory variables } \\
\hline Baseline FG (mmol/l) & $8.97(3.86)$ & $7.53(2.54)$ & $8.83(3.40)$ & $10.54(4.71)$ & $<0.001$ \\
\hline Mean 12-month FG & $8.10(2.42)$ & $6.90(1.46)$ & $7.98(2.04)$ & $9.35(2.78)$ & $<0.001$ \\
\hline Mean 24-month FG & $8.09(2.39)$ & $6.93(1.47)$ & $7.97(2.07)$ & $9.35(2.78)$ & $<0.001$ \\
\hline Baseline $\mathrm{HbA}_{1 \mathrm{c}}(\%)$ & $8.1(1.9)$ & $7.0(1.3)$ & $8.0(1.6)$ & $9.1(2.1)$ & $<0.001$ \\
\hline$(\mathrm{mmol} / \mathrm{mol})$ & $65(20.8)$ & $53(14.2)$ & $64(17.5)$ & $76(23.0)$ & \\
\hline Mean 12-month $\mathrm{HbA}_{1 \mathrm{c}}(\%)$ & $7.8(1.5)$ & $6.8(0.7)$ & $7.7(1.2)$ & $9.0(1.4)$ & $<0.001$ \\
\hline$(\mathrm{mmol} / \mathrm{mol})$ & $62(16.4)$ & $51(7.7)$ & $61(13.1)$ & $75(15.3)$ & \\
\hline Mean 24-month $\mathrm{HbA}_{1 \mathrm{c}}(\%)$ & $7.8(1.4)$ & $6.8(0.8)$ & $7.7(1.2)$ & $9.0(1.4)$ & $<0.001$ \\
\hline$(\mathrm{mmol} / \mathrm{mol})$ & $62(15.3)$ & $51(8.7)$ & $61(13.1)$ & $75(15.3)$ & \\
\hline Triacylglycerol (mmol/l) & $2.0(1.5)$ & $1.8(1.2)$ & $2.0(1.6)$ & $2.2(1.6)$ & 0.064 \\
\hline HDL-cholesterol (mmol/l) & $1.11(0.30)$ & $1.10(0.27)$ & $1.12(0.33)$ & $1.11(0.32)$ & 0.79 \\
\hline LDL-cholesterol (mmol/l) & $3.03(1.00)$ & $2.96(0.91)$ & $3.00(1.08)$ & $3.13(0.99)$ & 0.20 \\
\hline Glomerular filtration rate $\left(\mathrm{ml} / \mathrm{min} / 1.73 \mathrm{~m}^{2}\right)$ & $81(20)$ & $80(19)$ & $82(20)$ & $80(22)$ & 0.53 \\
\hline Albuminuria (mg/24 h) & $13(7-42)$ & $10(6-22)$ & $13(7-41)$ & $19(8-90)$ & $<0.001$ \\
\hline
\end{tabular}


Table 1 (continued)

\begin{tabular}{|c|c|c|c|c|c|}
\hline Characteristics & $\begin{array}{l}\text { All patients } \\
(\mathrm{n}=654)\end{array}$ & $\begin{array}{l}\text { 1st-tertile } \\
\mathrm{HbA}_{1 \mathrm{c}}-\mathrm{SD} \\
\leq 0.45 \% \\
(\mathrm{n}=218)\end{array}$ & $\begin{array}{l}\text { 2nd-tertile } \\
\mathrm{HbA}_{1 \mathrm{c}}-\mathrm{SD} \\
0.46-0.85 \% \\
(\mathrm{n}=218)\end{array}$ & $\begin{array}{l}\text { 3rd-tertile } \\
\mathrm{HbA}_{1 \mathrm{c}}-\mathrm{SD} \\
\geq 0.86 \% \\
(\mathrm{n}=218)\end{array}$ & $\mathrm{p}$ value \\
\hline \multicolumn{6}{|l|}{ Macrovascular outcomes ${ }^{a}$} \\
\hline Total CVEs & $128(2.56)$ & $36(2.20)$ & $34(1.99)$ & $58(3.67)$ & 0.005 \\
\hline Major CVEs & $96(1.86)$ & $24(1.42)$ & $24(1.38)$ & $48(2.93)$ & 0.001 \\
\hline Cardiovascular mortality & $67(1.26)$ & $16(0.92)$ & $15(0.85)$ & $36(2.07)$ & 0.002 \\
\hline All-cause mortality & $158(2.97)$ & $44(2.52)$ & $49(2.77)$ & $65(3.74)$ & 0.10 \\
\hline \multicolumn{6}{|l|}{ Microvascular outcomes ${ }^{b}$} \\
\hline Retinopathy (incident/worsening) $(n=533)$ & $152(4.88)$ & $30(2.63)$ & $50(4.89)$ & $72(7.96)$ & $<0.001$ \\
\hline Renal composite $(n=615)$ & $183(4.71)$ & $54(4.11)$ & $47(3.63)$ & $82(6.58)$ & $<0.001$ \\
\hline Microalbuminuria (incident) $(n=436)$ & $89(3.23)$ & $31(2.88)$ & $25(2.80)$ & $33(4.26)$ & 0.21 \\
\hline Renal failure $(n=615)$ & $91(2.15)$ & $19(1.35)$ & $24(1.72)$ & $48(3.42)$ & $<0.001$ \\
\hline Peripheral neuropathy (incident/worsening) $(n=471)$ & $96(20.4 \%)$ & $21(13.5 \%)$ & $35(22.0 \%)$ & $40(25.5 \%)$ & 0.011 \\
\hline Peripheral neuropathy (incident) $(n=338)$ & $42(12.4 \%)$ & $11(9.4 \%)$ & $9(8.2 \%)$ & $22(19.8 \%)$ & 0.005 \\
\hline
\end{tabular}

Values are proportions, and means (standard deviations) or medians (interquartile range)

$H b A_{1 c}$ glycated hemoglobin, $A C E$ angiotensin-converting enzyme, $A R$ angiotensin II receptor, $S B P$ systolic blood pressure, $D B P$ diastolic blood pressure, $F G$ fasting glycemia, $H D L$ high-density lipoprotein, $L D L$ low-density lipoprotein, CVEs cardiovascular events

a Values are absolute numbers (incidence rate per 100 patient-years of follow-up)

b Values are absolute numbers (incidence rate per 100 patient-years of follow-up), except for peripheral neuropathy that are absolute numbers (proportions)

of outcomes. However, for all-cause and cardiovascular mortalities, FG variability appeared a stronger risk predictor than $\mathrm{HbA}_{1 \mathrm{c}}$ variability parameters. Regarding microvascular outcomes, no glycemic variability parameter predicted diabetic retinopathy incidence or worsening, which associations were mostly attenuated by mean $\mathrm{HbA}_{1 \mathrm{c}}$ levels. Excluding patients with pre-existent retinopathy (i.e., analyzing separately only new retinopathy development) did not change these results. For renal outcomes, only 24-month FG variability predicted new microalbuminuria development, whereas both $\mathrm{HbA}_{1 \mathrm{c}}$ and FG variabilities independently predicted renal failure outcomes. Excluding patients with nephropathy at baseline (albuminuria $\geq 30 \mathrm{mg} / 24 \mathrm{~h}$ or eGFR $<60 \mathrm{ml} /$ $\min / 1.73 \mathrm{~m}^{2}$ ) also did not change any of the results of renal outcomes. Otherwise, substituting baseline nephropathy by its individual components (albuminuria and eGFR as continuous variables) as adjusting covariates widened the confidence intervals towards non-significant values; although there were only slight reductions in HRs. $\mathrm{HbA}_{1 \mathrm{c}}$ variability predicted new-development of diabetic peripheral neuropathy, but not its worsening. Otherwise, mean $\mathrm{HbA}_{1 \mathrm{c}}$ levels were not predictors of any macrovascular and mortality outcomes, and were only predictors of retinopathy and peripheral neuropathy development or progression, but not of new neuropathy incidence. Tables 5 and 6 show the same analyses with patients categorized into tertiles of glycemic variability parameters, and the results were mainly consistent with the continuous parameters analyses, although some parameters were non-significant because of the wider confidence intervals associated with the lower number of endpoints in each tertile subgroups. There was evidence of interaction $(\mathrm{p}<0.10)$ between mean $\mathrm{HbA}_{1 \mathrm{c}}$ levels and glycemic variability in analyses for diabetic retinopathy. In stratified analyses, 24-month $\mathrm{HbA}_{1 \mathrm{c}}$ variability predicted retinopathy incidence or worsening in individuals with lower $\mathrm{HbA}_{1 \mathrm{c}}$ levels $(\leq 7.5 \%, 58 \mathrm{mmol} / \mathrm{mol})$, with HRs of $1.88(95 \%$ CI 1.03-3.43; $\mathrm{p}=0.039)$ for $\mathrm{HbA}_{1 \mathrm{c}}-\mathrm{SD}$ and 1.56 (95\% CI 1.03-2.36; $\mathrm{p}=0.036$ ) for $\mathrm{HbA}_{1 \mathrm{c}}-\mathrm{VC}$. Similarly, when divided into tertiles, the upper tertile of $\mathrm{HbA}_{1 \mathrm{c}}$-SD (HR 3.26; 95\% CI 1.24-8.52; $\left.\mathrm{p}=0.016\right)$ and of $\mathrm{HbA}_{1 \mathrm{c}}-\mathrm{VC}(\mathrm{HR} 2.70$; 95\% CI 1.15-6.32; $\mathrm{p}=0.023)$ also predicted retinopathy development or progression. Otherwise, in patients with higher HbA1c levels, no glycemic variability parameter was predictive of retinopathy. There 
Table 2 Characteristics and endpoints incidence of all diabetic patients and divided into tertiles of 24-month fasting glycemia variability

\begin{tabular}{|c|c|c|c|c|c|}
\hline Characteristics & $\begin{array}{l}\text { All patients } \\
(n=654)\end{array}$ & $\begin{array}{l}1 \text { st-tertile } \\
\text { FG-SD } \\
\leq 1.40 \mathrm{mmol} / \mathrm{l} \\
(\mathrm{n}=218)\end{array}$ & $\begin{array}{l}\text { 2nd-tertile } \\
\text { FG-SD } \\
1.41-2.60 \mathrm{mmol} / \mathrm{l} \\
(\mathrm{n}=218)\end{array}$ & $\begin{array}{l}\text { 3rd-tertile } \\
\text { FG-SD } \\
\geq 2.61 \mathrm{mmol} / \mathrm{l} \\
(\mathrm{n}=218)\end{array}$ & $p$ value \\
\hline Age (years) & $60.1(9.6)$ & $60.6(9.8)$ & $60.5(9.2)$ & $59.1(9.5)$ & 0.16 \\
\hline Male sex (\%) & 38.1 & 38.7 & 36.2 & 39.9 & 0.45 \\
\hline BMI $\left(\mathrm{kg} / \mathrm{m}^{2}\right)$ & $29.8(4.6)$ & $29.9(4.6)$ & $29.9(5.0)$ & $29.5(5.0)$ & 0.63 \\
\hline Smoking, current/past (\%) & 45.1 & 46.5 & 38.5 & 50.9 & 0.031 \\
\hline Physical activity (\%) & 22.4 & 27.6 & 20.2 & 18.9 & 0.017 \\
\hline Diabetes duration (years) & $8(3-15)$ & $4(1-10)$ & $10(5-15)$ & $10(5-17)$ & $<0.001$ \\
\hline \multicolumn{6}{|l|}{ Chronic diabetic complications (\%) } \\
\hline Cerebrovascular disease & 9.0 & 6.9 & 6.4 & 13.3 & 0.019 \\
\hline Coronary artery disease & 15.6 & 11.1 & 15.1 & 21.1 & 0.015 \\
\hline Peripheral artery disease & 17.0 & 10.6 & 19.9 & 20.6 & 0.008 \\
\hline Retinopathy & 32.7 & 19.2 & 36.4 & 41.4 & $<0.001$ \\
\hline Nephropathy & 31.0 & 22.4 & 26.0 & 45.8 & $<0.001$ \\
\hline Peripheral neuropathy & 29.0 & 20.4 & 30.4 & 37.3 & 0.001 \\
\hline Cardiovascular autonomic neuropathy & 18.1 & 12.4 & 21.2 & 21.4 & 0.042 \\
\hline \multicolumn{6}{|l|}{ Diabetes treatment (\%) } \\
\hline Metformin & 87.9 & 90.8 & 91.3 & 80.7 & 0.001 \\
\hline Sulfonylureas & 43.3 & 40.6 & 50.9 & 39.4 & 0.029 \\
\hline Insulin & 48.9 & 24.9 & 52.3 & 68.8 & $<0.001$ \\
\hline Aspirin & 90.9 & 87.5 & 90.2 & 94.5 & 0.040 \\
\hline Dyslipidemia (\%) & 87.3 & 88.0 & 87.2 & 86.7 & 0.92 \\
\hline Statins use (\%) & 77.5 & 78.2 & 77.9 & 76.0 & 0.84 \\
\hline Arterial hypertension (\%) & 86.5 & 83.9 & 89.0 & 86.6 & 0.29 \\
\hline Number of anti-hypertensive drugs & $3(1-4)$ & $2(1-3)$ & $3(2-4)$ & $3(1-4)$ & 0.001 \\
\hline ACE inhibitors/AR blockers (\%) & 83.0 & 80.6 & 84.2 & 84.8 & 0.47 \\
\hline Diuretics (\%) & 67.7 & 59.7 & 75.7 & 68.6 & 0.002 \\
\hline Calcium channel blockers (\%) & 31.7 & 26.7 & 35.6 & 34.3 & 0.11 \\
\hline Beta-blockers (\%) & 50.1 & 43.2 & 54.0 & 53.9 & 0.043 \\
\hline \multicolumn{6}{|l|}{ Blood pressures $(\mathrm{mmHg})$} \\
\hline Clinic SBP & $147(25)$ & $143(22)$ & $149(26)$ & $149(25)$ & 0.012 \\
\hline Clinic DBP & $84(13)$ & $83(12)$ & $85(14)$ & $85(14)$ & 0.11 \\
\hline Ambulatory 24 h SBP & $128(15)$ & $125(13)$ & $129(15)$ & $131(17)$ & 0.001 \\
\hline Ambulatory $24 \mathrm{~h} \mathrm{DBP}$ & $74(10)$ & $73(9)$ & $74(9)$ & $75(11)$ & 0.078 \\
\hline \multicolumn{6}{|l|}{ Laboratory variables } \\
\hline Baseline FG (mmol/l) & 8.99 (3.89) & $7.55(2.83)$ & $9.27(3.72)$ & $10.16(4.44)$ & $<0.001$ \\
\hline Mean 12-month FG & $8.10(2.42)$ & $6.77(1.67)$ & $7.94(2.11)$ & $9.55(2.45)$ & $<0.001$ \\
\hline Mean 24-month FG & $8.09(2.39)$ & $6.83(1.67)$ & $7.88(2.11)$ & $9.55(2.44)$ & $<0.001$ \\
\hline Baseline $\mathrm{HbA}_{1 \mathrm{c}}(\%)$ & $8.1(1.9)$ & $7.1(1.5)$ & $8.1(1.7)$ & $9.0(2.1)$ & $<0.001$ \\
\hline$(\mathrm{mmol} / \mathrm{mol})$ & $65(20.8)$ & $54(16.4)$ & 65 (18.6) & $75(23.0)$ & \\
\hline Mean 12-month $\mathrm{HbA}_{1 \mathrm{c}}(\%)$ & $7.8(1.5)$ & $6.9(1.0)$ & $7.7(1.1)$ & $8.9(1.5)$ & $<0.001$ \\
\hline$(\mathrm{mmol} / \mathrm{mol})$ & $62(16.4)$ & $52(10.9)$ & $61(12.0)$ & $74(16.4)$ & \\
\hline Mean 24-month $\mathrm{HbA}_{1 \mathrm{c}}(\%)$ & $7.8(1.4)$ & $6.9(0.9)$ & $7.7(1.1)$ & $8.9(1.4)$ & $<0.001$ \\
\hline$(\mathrm{mmol} / \mathrm{mol})$ & $62(15.3)$ & $52(9.8)$ & $61(12.0)$ & $74(15.3)$ & \\
\hline Triacylglycerol (mmol/l) & $2.0(1.5)$ & $1.9(1.4)$ & $1.9(1.2)$ & $2.3(1.8)$ & 0.081 \\
\hline HDL-cholesterol (mmol/l) & $1.11(0.30)$ & $1.11(0.28)$ & $1.11(0.28)$ & $1.09(0.34)$ & 0.47 \\
\hline LDL-cholesterol (mmol/l) & $3.03(1.00)$ & $3.10(1.01)$ & $2.97(0.93)$ & $3.05(1.09)$ & 0.48 \\
\hline Glomerular filtration rate $\left(\mathrm{ml} / \mathrm{min} / 1.73 \mathrm{~m}^{2}\right)$ & $81(20)$ & $82(18)$ & $81(20)$ & $79(23)$ & 0.22 \\
\hline
\end{tabular}


Table 2 (continued)

\begin{tabular}{|c|c|c|c|c|c|}
\hline Characteristics & $\begin{array}{l}\text { All patients } \\
(n=654)\end{array}$ & $\begin{array}{l}\text { 1st-tertile } \\
\text { FG-SD } \\
\leq 1.40 \mathrm{mmol} / \mathrm{l} \\
(\mathrm{n}=218)\end{array}$ & $\begin{array}{l}\text { 2nd-tertile } \\
\text { FG-SD } \\
1.41-2.60 \mathrm{mmol} / \mathrm{l} \\
(\mathrm{n}=218)\end{array}$ & $\begin{array}{l}\text { 3rd-tertile } \\
\text { FG-SD } \\
\geq 2.61 \mathrm{mmol} / \mathrm{l} \\
(\mathrm{n}=218)\end{array}$ & $\mathrm{p}$ value \\
\hline Albuminuria (mg/24 h) & $13(7-42)$ & $10(6-23)$ & $13(7-28)$ & $20(9-98)$ & $<0.001$ \\
\hline \multicolumn{6}{|l|}{ Macrovascular outcomes ${ }^{\mathrm{a}}$} \\
\hline Total CVEs & $128(2.56)$ & $29(1.69)$ & $39(2.38)$ & $60(3.89)$ & $<0.001$ \\
\hline Major CVEs & $96(1.86)$ & $21(1.20)$ & $28(1.67)$ & $47(2.92)$ & $<0.001$ \\
\hline Cardiovascular mortality & $67(1.26)$ & $14(0.77)$ & $20(1.17)$ & $33(1.94)$ & 0.007 \\
\hline All-cause mortality & $158(2.97)$ & $36(1.99)$ & $46(2.69)$ & $76(4.47)$ & $<0.001$ \\
\hline \multicolumn{6}{|l|}{ Microvascular outcomes ${ }^{\mathrm{b}}$} \\
\hline Retinopathy (incident/worsening) $(n=533)$ & $152(4.88)$ & $32(2.73)$ & $42(4.15)$ & $78(8.84)$ & $<0.001$ \\
\hline Renal composite $(n=615)$ & $183(4.71)$ & $43(3.16)$ & $57(4.64)$ & $83(6.71)$ & $<0.001$ \\
\hline Microalbuminuria (incident) $(n=436)$ & $89(3.23)$ & $25(2.30)$ & $33(3.61)$ & $31(4.30)$ & 0.048 \\
\hline Renal failure $(n=615)$ & $91(2.15)$ & $16(1.10)$ & $25(1.85)$ & $50(3.63)$ & $<0.001$ \\
\hline Peripheral neuropathy (incident/worsening) $(n=471)$ & $96(20.4 \%)$ & $20(12.1 \%)$ & $34(22.1 \%)$ & $42(28.4 \%)$ & $<0.001$ \\
\hline Peripheral neuropathy (incident) $(n=338)$ & $42(12.4 \%)$ & $13(9.7 \%)$ & $13(12.4 \%)$ & $16(16.8 \%)$ & 0.11 \\
\hline
\end{tabular}

Values are proportions, and means (standard deviations) or medians (interquartile range)

FG fasting glycemia, $A C E$ angiotensin-converting enzyme, $A R$ angiotensin II receptor, $S B P$ systolic blood pressure, $D B P$ diastolic blood pressure, $H b A_{1 c}$ glycated hemoglobin, $H D L$ high-density lipoprotein, $L D L$ low-density lipoprotein, CVEs cardiovascular events

${ }^{a}$ Values are absolute numbers (incidence rate per 100 patient-years of follow-up)

b Values are absolute numbers (incidence rate per 100 patient-years of follow-up), except for peripheral neuropathy that are absolute numbers (proportions)

was no evidence of interaction in any of the other outcomes (all $\mathrm{p}>0.15$ for interaction terms).

\section{Discussion}

This prospective cohort study with a median follow-up of nearly 10 years has some important new findings. First, it demonstrated that for all micro- and macrovascular outcomes, except for retinopathy and peripheral neuropathy development or progression, 24-month visit-tovisit glycemic variability parameters, either estimated for $\mathrm{HbA}_{1 \mathrm{c}}$ or for FG, were better risk predictors than mean $\mathrm{HbA}_{1 \mathrm{c}}$ levels during this same time interval. Second, specifically for diabetic retinopathy development or progression, 24-month $\mathrm{HbA}_{1 \mathrm{c}}$ variability was a significant risk predictor in patients with good glycemic control (with mean $\mathrm{HbA} 1 \mathrm{c} \leq 7.5 \%, 58 \mathrm{mmol} / \mathrm{mol}$ ); but not in those with poorer controlled diabetes (HbA1c > 7.5\%), where mean $\mathrm{HbA}_{1 \mathrm{c}}$ levels were the main risk predictor. Third, specifically for peripheral neuropathy, mean $\mathrm{HbA}_{1 \mathrm{c}}$ was the main risk predictor for the composite outcome of developing or worsening neuropathy, whereas $\mathrm{HbA}_{1 \mathrm{c}}$ variability was a better risk predictor for new-incident peripheral neuropathy. Overall, our findings support the concept that long-term visit-to-visit glycemic variability is an additional and frequently a better glycemic parameter than mean $\mathrm{HbA}_{1 \mathrm{c}}$ levels for assessing the risk of future development of micro- and macrovascular diabetic complications.

Several previous studies evaluated long-term glycemic variability parameters in patients with type 2 diabetes [13-41]. As a whole, most agree that glycemic variability predicts all-cause mortality [29, 32, 33, 36-40], fatal or non-fatal cardiovascular diseases [23, 28-32], new microalbuminuria development $[16,18-22,25]$ and renal function deterioration $[16,23,24,26]$, although there were opposing reports for these outcomes [19, 29, 33-36, 41]. A recent meta-analysis including studies published until 2014 confirmed these findings [12]. Our results support these previous investigations. An intriguing finding of our study was that, mainly for cardiovascular and all-cause mortalities, FG variability seemed a stronger risk predictor than $\mathrm{HbA}_{1 \mathrm{c}}$ variability. 


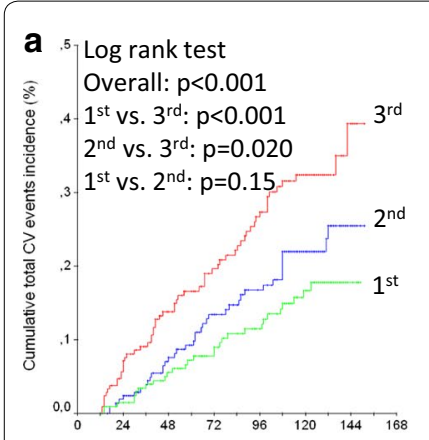

Number of patients at risk: $1^{\text {st. }}: 218201178151134 \quad 84 \quad 10$ 2nd: $218202173 \quad 141 \quad 118 \quad 77 \quad 7$ $3^{\text {rd: }} 218197157131112 \quad 69 \quad 11$

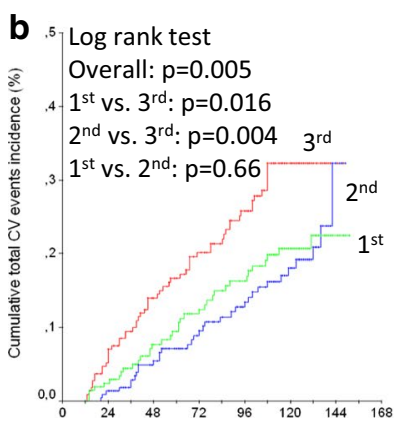

Number of patients at risk: $1^{\text {st. }} 218195166141123 \quad 81 \quad 10$ $2^{\text {nd: }}: 218211181148128 \quad 80 \quad 7$ $3^{\text {rd }}: 218200164135114 \quad 71 \quad 11$

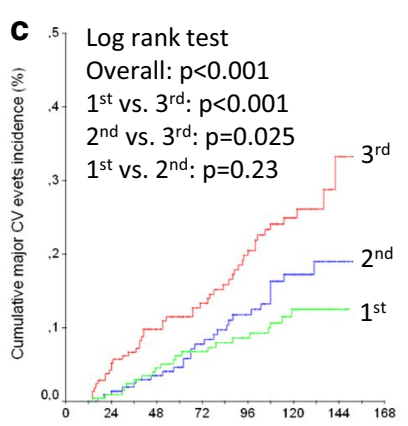

Number of patients at risk:

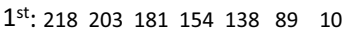

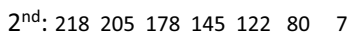
$3^{\text {rd: }} 218 \quad 200165139120 \quad 74 \quad 11$

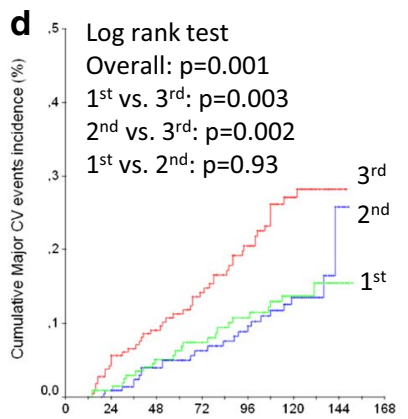

Number of patients at risk: $1^{\text {st. }}: 218 \quad 200172 \quad 146 \quad 128 \quad 87 \quad 10$ $2^{\text {nd: }}: 218212 \quad 183151132 \quad 85 \quad 7$ $3^{\text {rd }}: 218 \quad 202 \quad \begin{array}{llllll}173 & 143 & 122 & 73 & 11\end{array}$

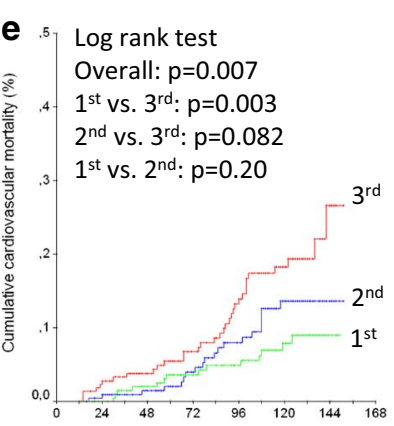

Number of patients at risk: $1^{\text {st. }} 218206186160145 \quad 95 \quad 10$ $2^{\text {nd: }: 218208181} 150126 \quad 82 \quad 8$ $3^{\text {rd: }} 218206176150130 \quad 81 \quad 12$

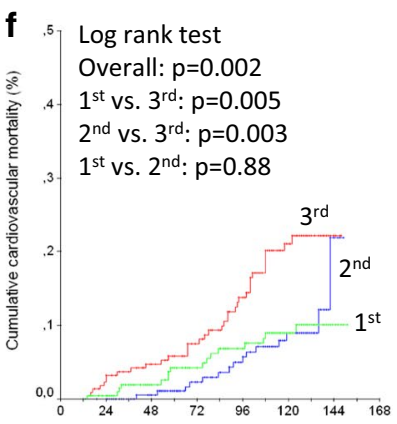

Number of patients at risk: $1^{\text {st: }}: 21820317815213593 \quad 11$ $2^{\text {nd: }} 218214187154135 \quad 87 \quad 7$ $3^{\text {rd }}: 218 \quad 209 \quad 183156 \quad 133 \quad 80 \quad 12$

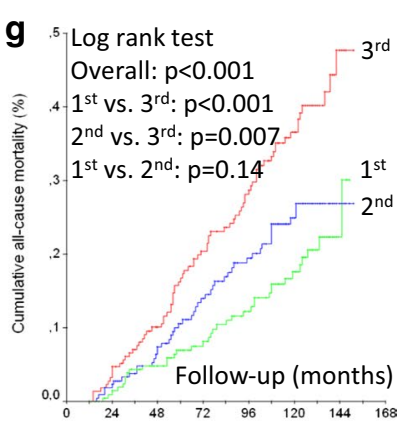

Number of patients at risk: $1^{\text {st }}: 218206186160145 \quad 95 \quad 10$ $2^{\text {nd }}: 218208181150126 \quad 82 \quad 8$ $3^{\text {rd: }} 218206176 \quad 150130 \quad 81 \quad 12$

h ${ }^{5}{ }^{5}$ Log rank test Overall: $p=0.10$

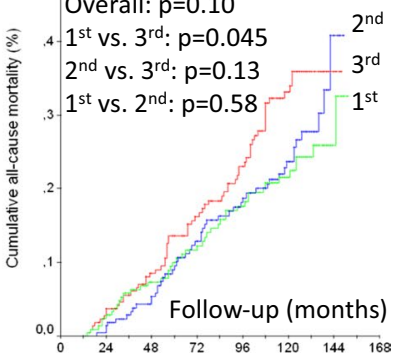

Number of patients at risk: $1^{\text {st: }} 218203178152135 \quad 93 \quad 11$ $2^{\text {nd: }} 218214187154135 \quad 87 \quad 7$ $3^{\text {rd: }} 218209183156133 \quad 80 \quad 12$

Fig. 1 Kaplan-Meier estimates of cumulative incidence of total cardiovascular events (a, b), major cardiovascular events (MACE, c, d), cardiovascular deaths $(\mathbf{e}, \mathbf{f})$ and all-cause deaths $(\mathbf{g}, \mathbf{h})$ during follow-up in patients categorized into tertiles (green curve first tertile, blue curve second tertile, and red curve third tertile) of 24-month fasting glycemia standard deviation (upper panels $\mathbf{a}, \mathbf{c}$, e and $\mathbf{g}$ ) and $\mathrm{HbA}_{1 \mathrm{c}}$ standard deviation (lower panels $\mathbf{b}$, $\mathbf{d}, \mathbf{f}$ and $\mathbf{h}$ )

The reason can be simple statistical adjustments because mean $\mathrm{HbA}_{1 \mathrm{c}}$ is expected to attenuate more $\mathrm{HbA}_{1 \mathrm{c}}$ variability than FG variability. However, models were also adjusted for mean FG levels that would attenuate FG variability, although not at the same extent given that mean $\mathrm{HbA}_{1 \mathrm{c}}$ was more strongly associated with the outcomes than mean FG. Alternatively, FG variability may have captured more accurately hypoglycemic episodes than $\mathrm{HbA}_{1 \mathrm{c}}$ variability [36, 48]. Severe hypoglycemia is well-known associated with adverse prognosis in type 2 diabetes, particularly with increased mortality $[49,50]$. Postprandial hyperglycemia is another issue of concern that might not have been adequately captured by either
FG or $\mathrm{HbA}_{1 \mathrm{c}}$ variability parameters. In this regard, low 1,5-anhydroglucitol levels, a potential marker of postprandial hyperglycemia, have been reported to predict worse cardiovascular outcomes in patients with acute coronary syndrome [51] and in patients with stable coronary heart disease submitted to elective angiography [52], both groups with low $\mathrm{HbA}_{1 \mathrm{c}}$ levels $(<7.0 \%)$.

Regarding the value of glycemic variability as risk predictor for future diabetic retinopathy development or progression, previous reports were controversial, with some showing the predictive capacity of FG variability $[13,15]$, whereas others negated the importance of glycemic variability parameters $[14,16-18]$. The recent 


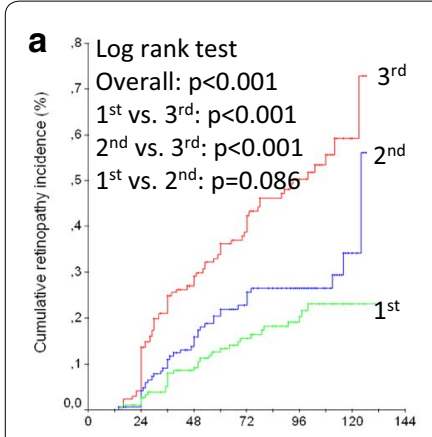

Number of patients at risk: $1^{\text {st: }} \begin{array}{llllll}186 & 181 & 143 & 109 & 73 & 8\end{array}$ $2^{\text {nd: }} \begin{array}{lllllll}174 & 168 & 123 & 84 & 61 & 7\end{array}$ $3^{\text {rd: }} \begin{array}{llllll}173 & 162 & 101 & 69 & 40 & 6\end{array}$

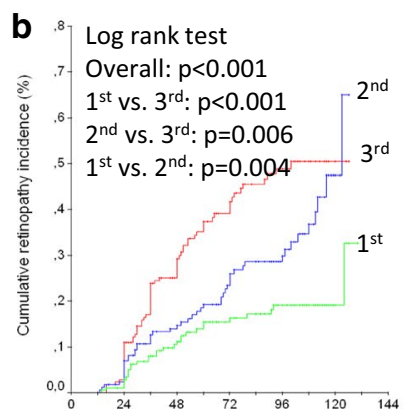

Number of patients at risk: $\begin{array}{lllllll}1^{\text {st: }} & 186 & 177 & 137 & 101 & 74 & 9\end{array}$ $2^{\text {nd: }} \begin{array}{llllll}174 & 168 & 121 & 91 & 60 & 6\end{array}$ $3^{\text {rd: }} \begin{array}{lllllll}173 & 168 & 109 & 69 & 40 & 6\end{array}$

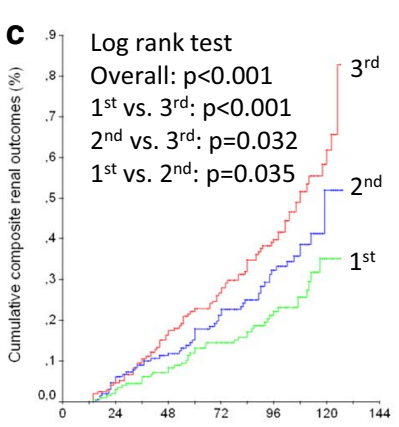

Number of patients at risk: $1^{\text {st: }} \begin{array}{llllll}202 & 195 & 163 & 132 & 86 & 9\end{array}$ $2^{\text {nd: }} \begin{array}{lllllll}198 & 182 & 146 & 112 & 69 & 8\end{array}$ $3^{\text {rd: }} \begin{array}{llllll}205 & 182 & 144 & 113 & 76 & 12\end{array}$

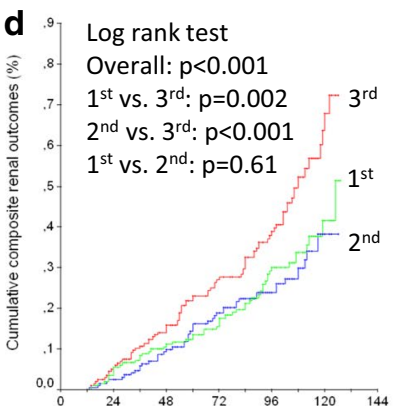

Number of patients at risk: $1^{\text {st: }} \quad \begin{array}{llllll}203 & 189 & 152 & 126 & 81 & 12\end{array}$ $2^{\text {nd }}: \begin{array}{llllll}202 & 190 & 153 & 121 & 79 & 8\end{array}$ $3^{\text {rd: }} \quad \begin{array}{llllll}210 & 186 & 150 & 111 & 73 & 9\end{array}$ e ${ }^{5}{ }^{5}$ Log rank test Overall: $p=0.048$

@ $.41^{\text {st }}$ vs. $3^{\text {rd }}: p=0.014$

$2^{\text {nd }}$ vs. $3^{\text {rd: }} p=0.50 \quad 3^{\text {rd }}$

的 $1^{\text {st }}$ vs. $2^{\text {nd }}: p=0.076$
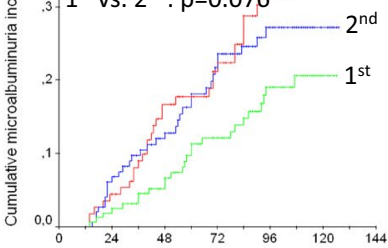

Number of patients at risk: $1^{\text {st: }} 159 \quad 156 \quad 131 \quad 106 \quad 69 \quad 8$ $2^{\text {nd: }} \begin{array}{lllllll}147 & 135 & 109 & 85 & 48 & 8\end{array}$

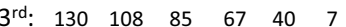

\section{f ${ }^{.5}$ Log rank test} Overall: $p=0.21$

. ${ }^{\circ}-1^{\text {st }}$ vs. $3^{\text {rd }}: p=0.11$

$\quad 2^{\text {nd }}$ vs. $3^{\text {rd }}: p=0.15 \quad 3^{\text {rd }}$

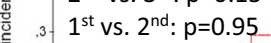

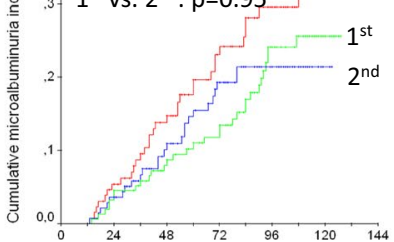

Number of patients at risk:

$1^{\text {st: }} \begin{array}{lllllll}159 & 150 & 126 & 109 & 67 & 12\end{array}$

$2^{\text {nd: }} \begin{array}{lllllll}147 & 133 & 104 & 83 & 55 & 4\end{array}$

$\begin{array}{lllllll}3^{\text {rd: }} & 130 & 120 & 97 & 67 & 36 & 7\end{array}$

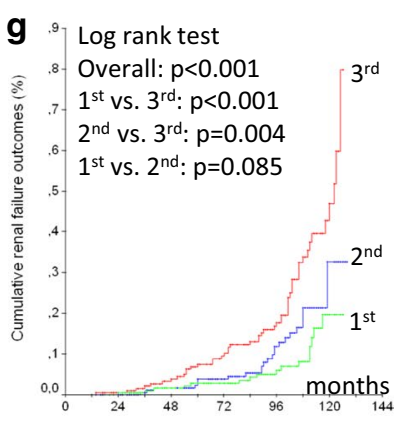

Number of patients at risk: $1^{\text {st: }} \quad \begin{array}{llllll}203 & 199 & 169 & 147 & 103 & 10\end{array}$ $2^{\text {nd: }} \begin{array}{lllllll}202 & 192 & 160 & 133 & 89 & 10\end{array}$

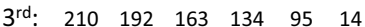

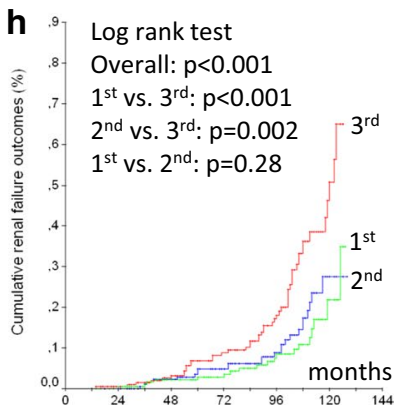

Number of patients at risk: $1^{\text {st: }} \quad \begin{array}{llllll}203 & 195 & 161 & 140 & 101 & 13\end{array}$ $2^{\text {nd: }} \begin{array}{llllll}202 & 199 & 163 & 139 & 94 & 9\end{array}$ $3^{\text {rd: }} \quad \begin{array}{llllll}210 & 196 & 170 & 136 & 94 & 12\end{array}$

Fig. 2 Kaplan-Meier estimates of cumulative diabetic retinopathy incidence or worsening $(\mathbf{a}, \mathbf{b})$, composite renal events (microalbuminuria and renal function deterioration, $\mathbf{c}, \mathbf{d})$, microalbuminuria incidence $(\mathbf{e}, \mathbf{f})$ and renal function deterioration $(\mathbf{g}, \mathbf{h})$ during follow-up in patients categorized into tertiles (green curve first tertile, blue curve second tertile, and red curve third tertile) of 24-month fasting glycemia standard deviation (upper panels $\mathbf{a}, \mathbf{c}$, e and $\mathbf{g}$ ) and of 24-month $\mathrm{HbA}_{1 \mathrm{c}}$ standard deviation (lower panels $\mathbf{b}, \mathbf{d}, \mathbf{f}$ and $\mathbf{h}$ )

meta-analysis also did not demonstrate any prognostic value for retinopathy development [12], but included only two studies. Our study provided new findings, by showing that the predictive power of $\mathrm{HbA}_{1 \mathrm{c}}$ variability for retinopathy development or progression depends on mean $\mathrm{HbA}_{1 \mathrm{c}}$ levels, being positive in patients with better-controlled diabetes, but absent in those poorly-controlled.

As far as we know, this is the first prospective study to assess the importance of glycemic variability for predicting diabetic peripheral neuropathy development or progression. Only a previous study [27] had evaluated its value for cardiovascular autonomic neuropathy development, with positive findings. We showed that
$\mathrm{HbA}_{1 \mathrm{c}}$ variability was a predictor mainly of new-incident peripheral neuropathy, whereas mean $\mathrm{HbA}_{1 \mathrm{c}}$ levels mainly predicted its worsening.

From a physiopathological standpoint, there were several potential mechanisms that may link increased glycemic variability to the future occurrence of diabetic micro- and macrovascular complications and to mortality. Acute, short-term glycemia fluctuations induce superoxide overproduction, increased oxidative stress, inflammatory cytokines generation and endothelial dysfunction and damage [11, 53, 54], all linked to chronic diabetic complications. Moreover, exaggerated glycemic fluctuations were demonstrated to adversely affect endothelial vessel healing, increasing neointimal 
Table 3 Results of Cox survival analyses for the excess risks associated with 12- and 24-month glycemic variability parameters, analyzed as continuous variables, for the occurrence of future macrovascular complications and mortality

\begin{tabular}{|c|c|c|c|c|c|c|c|}
\hline \multirow[t]{2}{*}{ Outcome } & \multirow{2}{*}{$\begin{array}{l}\text { Glycemic } \\
\text { parameter }\end{array}$} & \multicolumn{3}{|c|}{ 12-month glycemic variability } & \multicolumn{3}{|c|}{ 24-month glycemic variability } \\
\hline & & $\begin{array}{l}\text { Model } 1 \\
\text { HR (95\% Cl) }\end{array}$ & $\begin{array}{l}\text { Model } 2 \\
\text { HR }(95 \% \text { Cl) }\end{array}$ & $\begin{array}{l}\text { Model } 3 \\
\text { HR }(95 \% \mathrm{Cl})\end{array}$ & $\begin{array}{l}\text { Model } 1 \\
\text { HR }(95 \% \mathrm{Cl})\end{array}$ & $\begin{array}{l}\text { Model } 2 \\
\text { HR }(95 \% \mathrm{Cl})\end{array}$ & $\begin{array}{l}\text { Model } 3 \\
\text { HR }(95 \% \mathrm{Cl})\end{array}$ \\
\hline \multirow{5}{*}{$\begin{array}{l}\text { Total CV events } \\
n=128\end{array}$} & FG-SD & $1.48(1.28-1.70)^{*}$ & $1.30(1.10-1.54)^{\dagger}$ & $1.23(1.00-1.51)$ & $1.54(1.34-1.78)^{*}$ & $1.41(1.18-1.67)^{*}$ & $1.36(1.09-1.69)^{\dagger}$ \\
\hline & FG-VC & $1.48(1.25-1.74)^{*}$ & $1.33(1.10-1.61)^{\dagger}$ & $1.26(1.04-1.54)^{\ddagger}$ & $1.58(1.33-1.88)^{*}$ & $1.44(1.18-1.75)^{*}$ & $1.37(1.12-1.69)^{\dagger}$ \\
\hline & $\mathrm{HbA}_{1 c}-\mathrm{SD}$ & $1.33(1.15-1.53)^{*}$ & $1.22(1.05-1.42)^{\dagger}$ & $1.11(0.91-1.34)$ & $1.39(1.21-1.60)^{*}$ & $1.29(1.11-1.49)^{\dagger}$ & $1.20(1.00-1.44)$ \\
\hline & $\mathrm{HbA}_{1 c}-\mathrm{VC}$ & $1.26(1.09-1.46)^{\dagger}$ & $1.19(1.03-1.38)^{\ddagger}$ & $1.10(0.93-1.30)$ & $1.32(1.14-1.52)^{*}$ & $1.25(1.08-1.44)^{\dagger}$ & $1.17(0.99-1.38)$ \\
\hline & 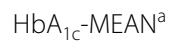 & - & - & $1.24(0.96-1.60)$ & - & - & $1.18(0.93-1.49)$ \\
\hline \multirow{5}{*}{$\begin{array}{l}\text { Major CV events } \\
n=96\end{array}$} & FG-SD & $1.51(1.28-1.77)^{*}$ & $1.33(1.09-1.61)^{\dagger}$ & $1.19(0.94-1.51)$ & $1.54(1.30-1.82)^{*}$ & $1.35(1.10-1.65)^{\dagger}$ & $1.23(0.96-1.59)$ \\
\hline & FG-VC & $1.46(1.20-1.77)^{*}$ & $1.31(1.05-1.63)^{\ddagger}$ & $1.24(0.99-1.57)$ & $1.53(1.25-1.87)^{*}$ & $1.34(1.06-1.69)^{\ddagger}$ & $1.29(1.01-1.64)^{\ddagger}$ \\
\hline & $\mathrm{HbA}_{1 c}-\mathrm{SD}$ & $1.40(1.19-1.63)^{*}$ & $1.28(1.08-1.51)^{\dagger}$ & $1.19(0.97-1.46)$ & $1.44(1.23-1.68)^{*}$ & $1.31(1.11-1.55)^{\dagger}$ & $1.23(1.00-1.51)^{\ddagger}$ \\
\hline & $\mathrm{HbA}_{1 c}-\mathrm{VC}$ & $1.34(1.14-1.57)^{*}$ & $1.25(1.06-1.47)^{\dagger}$ & $1.17(0.98-1.41)$ & $1.37(1.17-1.61)^{*}$ & $1.28(1.08-1.50)^{\dagger}$ & $1.21(1.01-1.44)^{\ddagger}$ \\
\hline & 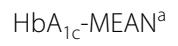 & - & - & $1.14(0.85-1.53)$ & - & - & $1.16(0.88-1.52)$ \\
\hline \multirow{5}{*}{$\begin{array}{l}\text { CV mortality } \\
n=67\end{array}$} & FG-SD & $1.51(1.26-1.82)^{*}$ & $1.47(1.17-1.86)^{*}$ & $1.29(0.97-1.73)$ & $1.65(1.36-2.00)^{*}$ & $1.64(1.29-2.09)^{*}$ & $1.50(1.10-2.03)^{\dagger}$ \\
\hline & FG-VC & $1.51(1.21-1.89)^{*}$ & $1.48(1.14-1.92)^{\dagger}$ & $1.37(1.04-1.81)^{\ddagger}$ & $1.70(1.34-2.16)^{*}$ & $1.64(1.25-2.15)^{*}$ & $1.56(1.17-2.06)^{\dagger}$ \\
\hline & $\mathrm{HbA}_{1 c}-\mathrm{SD}$ & $1.51(1.27-1.80)^{*}$ & $1.38(1.14-1.67)^{*}$ & $1.24(0.97-1.58)$ & $1.54(1.29-1.85)^{*}$ & $1.42(1.17-1.72)^{*}$ & $1.26(0.99-1.61)$ \\
\hline & $\mathrm{HbA}_{1 c}-\mathrm{VC}$ & $1.43(1.20-1.71)^{*}$ & $1.35(1.12-1.62)^{\dagger}$ & $1.23(1.00-1.52)^{\ddagger}$ & $1.44(1.21-1.73)^{*}$ & $1.37(1.13-1.65)^{*}$ & $1.24(1.01-1.54)^{\ddagger}$ \\
\hline & $\mathrm{HbA}_{1 c^{-M E A N}}{ }^{\mathrm{a}}$ & - & - & $1.28(0.88-1.84)$ & - & - & $1.27(0.91-1.79)$ \\
\hline \multirow{5}{*}{$\begin{array}{l}\text { All-cause mortality } \\
\mathrm{n}=158\end{array}$} & FG-SD & $1.51(1.34-1.71)^{*}$ & $1.42(1.23-1.65)^{*}$ & $1.44(1.20-1.73)^{*}$ & $1.60(1.41-1.81)^{*}$ & $1.54(1.32-1.79)^{*}$ & $1.59(1.32-1.93)^{*}$ \\
\hline & FG-VC & $1.53(1.33-1.77)^{*}$ & $1.45(1.24-1.71)^{*}$ & $1.43(1.20-1.69)^{*}$ & $1.66(1.42-1.93)^{*}$ & $1.55(1.31-1.85)^{*}$ & $1.53(1.28-1.82)^{*}$ \\
\hline & $\mathrm{HbA}_{1 c}-\mathrm{SD}$ & $1.41(1.25-1.60)^{*}$ & $1.29(1.13-1.48)^{*}$ & $1.25(1.06-1.47)^{\dagger}$ & $1.42(1.25-1.61)^{*}$ & $1.30(1.14-1.49)^{*}$ & $1.23(1.04-1.46)^{\ddagger}$ \\
\hline & $\mathrm{HbA}_{1 c}-\mathrm{VC}$ & $1.35(1.19-1.52)^{*}$ & $1.26(1.11-1.44)^{*}$ & $1.21(1.04-1.40)^{\ddagger}$ & $1.34(1.18-1.52)^{*}$ & $1.25(1.10-1.43)^{*}$ & $1.19(1.02-1.38)^{\ddagger}$ \\
\hline & $\mathrm{HbA}_{1 c^{-M E A N}}{ }^{\mathrm{a}}$ & - & - & $1.07(0.84-1.35)$ & - & - & $1.08(0.87-1.34)$ \\
\hline
\end{tabular}

Values are hazard ratios and $95 \%$ confidence intervals, estimated for increases of 1-SD in each glycemic parameter

Model 1 is adjusted for age, sex and number of $\mathrm{HbA}_{1 \mathrm{c}}$ or $\mathrm{FG}$ measurements

Model 2 is further adjusted for diabetes duration, BMI, smoking status, physical inactivity, arterial hypertension, number of anti-hypertensive drugs in use, ambulatory 24-h SBP, presence of micro- and macrovascular complications at baseline, serum mean HDL- and LDL-cholesterol, and use of insulin, statins and aspirin Model 3 is further adjusted for mean fasting glycemia and $\mathrm{HbA}_{1 \mathrm{c}}$

$H R$ hazard ratio, $C l$ confidence interval, $C V$ cardiovascular, $F G-S D$ fasting glucose standard deviation, $F G-V C$ fasting glucose variation coefficient, $H b A_{1 c}-S D$ glycated hemoglobin standard deviation, $H b A_{1 c}-V C$ glycated hemoglobin variation coefficient, $H b A_{1 c}-M E A N$ mean glycated hemoglobin during the same time interval ${ }^{*} p<0.001 ;{ }^{\dagger} p<0.01{ }^{\ddagger} p<0.05$

${ }^{a}$ The $\mathrm{HR}$ of $\mathrm{HbA}_{1 \mathrm{c}}-\mathrm{MEAN}$ was estimated also for increases of 1-SD in the model with the highest $\mathrm{HR}$ of the glycemic variability parameter, whichever it was

thickness following percutaneous stent implantation [55], and augmenting the risk of periprocedural and short-term cardiovascular complications [56, 57], which may be particularly important in such a high cardiovascular risk population as ours. Further, transient hyperglycemia might cause epigenetic changes, inducing cellular metabolic memory $[58,59]$, increasing insulin resistance [60] and pancreatic $\beta$-cell dysfunction and apoptosis [61]. Alternatively, but not excluding, increased glycemic variability might simply be a marker of unstable glycemic control due to poor treatment adherence and self-management patient compliance [12, 31], multimorbidity, poor quality of life and lack of social support, and frequent infections complications [12]. In this regard, it should be noted that patients with higher glycemic variability at baseline were less physically active, more frequently current or past smokers, and had a greater prevalence of diabetic complications than those with lower variability, particularly evident for FG variability.

The study has some limitations that shall be noted. First, it is a prospective observational cohort; hence no cause-and-effect relations, nor physiopathological 
Table 4 Results of multivariable analyses for the excess risks associated with 12- and 24-month glycemic variability parameters, analyzed as continuous variables, for the occurrence of future diabetic microvascular complications

\begin{tabular}{|c|c|c|c|c|c|c|c|}
\hline \multirow[t]{2}{*}{ Outcome } & \multirow{2}{*}{$\begin{array}{l}\text { Glycemic } \\
\text { parameter }\end{array}$} & \multicolumn{3}{|c|}{ 12-month glycemic variability } & \multicolumn{3}{|c|}{ 24-month glycemic variability } \\
\hline & & $\begin{array}{l}\text { Model } 1 \\
\text { HR (95\% CI) }\end{array}$ & $\begin{array}{l}\text { Model } 2 \\
\text { HR (95\% Cl) }\end{array}$ & $\begin{array}{l}\text { Model } 3 \\
\text { HR (95\% Cl) }\end{array}$ & $\begin{array}{l}\text { Model } 1 \\
\text { HR (95\% Cl) }\end{array}$ & $\begin{array}{l}\text { Model } 2 \\
\text { HR (95\% Cl) }\end{array}$ & $\begin{array}{l}\text { Model } 3 \\
\text { HR }(95 \% \mathrm{Cl})\end{array}$ \\
\hline \multirow{5}{*}{$\begin{array}{l}\text { Retinopathy } \\
\text { (incident or } \\
\text { worsening) } \\
n=152\end{array}$} & FG-SD & $1.54(1.35-1.76)^{*}$ & $1.27(1.08-1.49)^{\dagger}$ & $1.12(0.93-1.35)$ & $1.49(1.31-1.69)^{*}$ & $1.28(1.09-1.51)^{\dagger}$ & $1.11(0.91-1.35)$ \\
\hline & FG-VC & $1.48(1.27-1.71)^{*}$ & $1.18(1.00-1.39)^{\ddagger}$ & $1.12(0.94-1.34)$ & $1.48(1.27-1.72)^{*}$ & $1.20(1.01-1.43)^{\ddagger}$ & $1.13(0.93-1.36)$ \\
\hline & $\mathrm{HbA}_{1 c}-\mathrm{SD}$ & $1.27(1.12-1.43)^{*}$ & $1.16(1.00-1.33)^{\ddagger}$ & $0.99(0.83-1.18)$ & $1.31(1.16-1.47)^{*}$ & $1.21(1.05-1.39)^{\dagger}$ & $1.05(0.89-1.25)$ \\
\hline & $\mathrm{HbA}_{1 c}-\mathrm{VC}$ & $1.21(1.06-1.38)^{\dagger}$ & $1.12(0.97-1.30)$ & $1.00(0.85-1.18)$ & $1.27(1.11-1.44)^{*}$ & $1.17(1.02-1.34)^{\ddagger}$ & $1.06(0.91-1.24)$ \\
\hline & 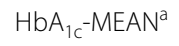 & - & - & $1.27(1.00-1.60)^{\ddagger}$ & - & - & $1.27(1.03-1.58)^{\ddagger}$ \\
\hline \multirow{5}{*}{$\begin{array}{l}\text { Composite renal } \\
\text { outcome } \\
n=183\end{array}$} & FG-SD & $1.29(1.14-1.47)^{*}$ & $1.19(1.03-1.39)^{\ddagger}$ & $1.15(0.97-1.36)$ & $1.37(1.20-1.55)^{*}$ & $1.30(1.12-1.51)^{\dagger}$ & $1.25(1.05-1.48)^{\ddagger}$ \\
\hline & FG-VC & $1.24(1.08-1.42)^{\dagger}$ & $1.14(0.98-1.33)$ & $1.11(0.94-1.30)$ & $1.30(1.13-1.49)^{*}$ & $1.21(1.04-1.41)^{\ddagger}$ & $1.19(1.01-1.40)^{\ddagger}$ \\
\hline & $\mathrm{HbA}_{1 c}-\mathrm{SD}$ & $1.24(1.09-1.40)^{\dagger}$ & $1.14(0.99-1.30)$ & $1.06(0.90-1.26)$ & $1.29(1.14-1.46)^{*}$ & $1.19(1.04-1.37)^{\ddagger}$ & $1.12(0.95-1.32)$ \\
\hline & $\mathrm{HbA}_{1 c}-\mathrm{VC}$ & $1.19(1.05-1.36)^{\dagger}$ & $1.11(0.97-1.27)$ & $1.05(0.90-1.22)$ & $1.23(1.09-1.40)^{\dagger}$ & $1.15(1.01-1.31)^{\ddagger}$ & $1.09(0.94-1.26)$ \\
\hline & $\mathrm{HbA}_{1 c^{-}}-\mathrm{MEAN}^{\mathrm{a}}$ & - & - & $1.15(0.91-1.46)$ & - & - & $1.12(0.91-1.39)$ \\
\hline \multirow{5}{*}{$\begin{array}{l}\text { Microalbuminuria } \\
\quad \text { (incident) } \\
n=89\end{array}$} & FG-SD & $1.21(0.98-1.48)$ & $1.24(0.98-1.56)$ & $1.21(0.91-1.62)$ & $1.27(1.04-1.56)^{\ddagger}$ & $1.34(1.06-1.69)^{\ddagger}$ & $1.34(1.00-1.79)^{\ddagger}$ \\
\hline & FG-VC & $1.14(0.93-1.41)$ & $1.17(0.93-1.48)$ & $1.15(0.90-1.48)$ & $1.21(0.98-1.48)$ & $1.26(1.00-1.58)^{\ddagger}$ & $1.25(0.98-1.60)$ \\
\hline & $\mathrm{HbA}_{1 c}-\mathrm{SD}$ & $1.13(0.92-1.40)$ & $1.11(0.89-1.39)$ & $1.02(0.76-1.37)$ & $1.20(0.98-1.47)$ & $1.19(0.96-1.47)$ & $1.12(0.84-1.49)$ \\
\hline & $\mathrm{HbA}_{1 \mathrm{c}}-\mathrm{VC}$ & $1.11(0.89-1.37)$ & $1.08(0.87-1.35)$ & $1.01(0.78-1.31)$ & $1.17(0.95-1.43)$ & $1.15(0.93-1.42)$ & $1.08(0.85-1.38)$ \\
\hline & $\mathrm{HbA}_{1 c^{-M E A N}}{ }^{\mathrm{a}}$ & - & - & $1.03(0.74-1.45)$ & - & - & $1.00(0.73-1.37)$ \\
\hline \multirow{5}{*}{$\begin{array}{l}\text { Renal failure } \\
n=91\end{array}$} & FG-SD & $1.54(1.31-1.83)^{*}$ & $1.27(1.05-1.55)^{\ddagger}$ & $1.22(0.98-1.53)$ & $1.70(1.44-2.01)^{*}$ & $1.43(1.16-1.77)^{\dagger}$ & $1.37(1.08-1.74)^{\dagger}$ \\
\hline & FG-VC & $1.50(1.24-1.82)^{*}$ & $1.25(1.01-1.53)^{\ddagger}$ & $1.18(0.95-1.46)$ & $1.64(1.34-2.00)^{*}$ & $1.32(1.06-1.64)^{\ddagger}$ & $1.29(1.02-1.61)^{\ddagger}$ \\
\hline & $\mathrm{HbA}_{1 c}-\mathrm{SD}$ & $1.51(1.29-1.76)^{*}$ & $1.34(1.12-1.60)^{\dagger}$ & $1.27(1.02-1.58)^{\ddagger}$ & $1.53(1.30-1.79)^{*}$ & $1.35(1.12-1.63)^{\dagger}$ & $1.25(1.00-1.60)^{\ddagger}$ \\
\hline & $\mathrm{HbA}_{1 c}-\mathrm{VC}$ & $1.43(1.22-1.68)^{*}$ & $1.33(1.11-1.59)^{\dagger}$ & $1.26(1.03-1.53)^{\ddagger}$ & $1.43(1.22-1.68)^{*}$ & $1.31(1.09-1.57)^{\dagger}$ & $1.23(1.01-1.50)^{\ddagger}$ \\
\hline & $\mathrm{HbA}_{1 c^{-}}-\mathrm{MEAN}^{\mathrm{a}}$ & - & - & $1.20(0.85-1.70)$ & - & - & $1.28(0.96-1.70)$ \\
\hline \multirow{5}{*}{$\begin{array}{l}\text { Peripheral neu- } \\
\text { ropathy (incident } \\
\text { or worsening) } \\
n=96\end{array}$} & FG-SD & $1.37(1.10-1.71)^{\dagger}$ & $1.09(0.82-1.44)$ & $0.96(0.69-1.34)$ & $1.58(1.25-2.00)^{*}$ & $1.31(0.98-1.76)$ & $1.21(0.86-1.70)$ \\
\hline & FG-VC & $1.38(1.10-1.73)^{\dagger}$ & $1.16(0.87-1.54)$ & $1.04(0.77-1.40)$ & $1.56(1.24-1.96)^{*}$ & $1.38(1.04-1.83)^{\ddagger}$ & $1.25(0.93-1.68)$ \\
\hline & $\mathrm{HbA}_{1 c}-\mathrm{SD}$ & $1.42(1.15-1.76)^{\dagger}$ & $1.30(1.01-1.66)^{\ddagger}$ & $1.11(0.82-1.50)$ & $1.51(1.21-1.87)^{*}$ & $1.37(1.07-1.75)^{\ddagger}$ & $1.17(0.86-1.60)$ \\
\hline & $\mathrm{HbA}_{1 c}-\mathrm{VC}$ & $1.33(1.07-1.65)^{\ddagger}$ & $1.22(0.95-1.55)$ & $1.08(0.83-1.42)$ & $1.41(1.14-1.74)^{\dagger}$ & $1.26(0.99-1.61)$ & $1.12(0.86-1.47)$ \\
\hline & $\mathrm{HbA}_{1 c^{-}} \mathrm{MEAN}^{\mathrm{a}}$ & - & - & $1.55(1.03-2.32)^{\ddagger}$ & - & - & $1.53(1.09-2.15)^{\ddagger}$ \\
\hline \multirow{5}{*}{$\begin{array}{l}\text { Peripheral neurop- } \\
\text { athy (incident) } \\
n=42\end{array}$} & FG-SD & $1.33(0.96-1.84)$ & $1.10(0.75-1.63)$ & $0.95(0.60-1.50)$ & $1.44(1.03-2.01)^{\ddagger}$ & $1.23(0.83-1.84)$ & $1.09(0.68-1.74)$ \\
\hline & FG-VC & $1.26(0.91-1.75)$ & $1.12(0.76-1.65)$ & $1.03(0.68-1.55)$ & $1.31(0.95-1.82)$ & $1.20(0.81-1.77)$ & $1.12(0.74-1.69)$ \\
\hline & $\mathrm{HbA}_{1 c}-\mathrm{SD}$ & $1.66(1.27-2.17)^{*}$ & $1.63(1.19-2.22)^{\dagger}$ & $1.73(1.15-2.60)^{\dagger}$ & $1.76(1.33-2.32)^{*}$ & $1.67(1.22-2.27)^{*}$ & $1.82(1.20-2.75)^{\dagger}$ \\
\hline & $\mathrm{HbA}_{1 c}-\mathrm{VC}$ & $1.63(1.25-2.14)^{*}$ & $1.58(1.16-2.16)^{\dagger}$ & $1.55(1.09-2.20)^{\ddagger}$ & $1.72(1.30-2.26)^{*}$ & $1.62(1.18-2.21)^{\dagger}$ & $1.60(1.12-2.28)^{\dagger}$ \\
\hline & $\mathrm{HbA}_{1 c^{-}} \mathrm{MEAN}^{\mathrm{a}}$ & - & - & $0.91(0.47-1.74)$ & - & - & $0.85(0.47-1.57)$ \\
\hline
\end{tabular}

Values are hazard ratios and 95\% confidence intervals, estimated by Cox analyses for increases of 1-SD in each glycemic parameter; except for peripheral neuropathy outcomes that are odds ratios and $95 \%$ confidence intervals, estimated by logistic regressions

Models 1, 2 and 3 were adjusted for the same covariates as in Table 3, except for peripheral neuropathy that was adjusted for height instead of BMI and further for the time interval between baseline and second neuropathy examination

$H R$ hazard ratio, $C l$ confidence interval, $F G$-SD fasting glucose standard deviation, $F G-V C$ fasting glucose variation coefficient, $H b A_{1 c}-S D$ glycated hemoglobin standard deviation, $H b A_{1 c}-V C$ glycated hemoglobin variation coefficient, $H b A_{1 c}-M E A N$ mean glycated hemoglobin during the same time interval

${ }^{*} \mathrm{p}<0.001 i^{\dagger} \mathrm{p}<0.01{ }^{\ddagger} \mathrm{p}<0.05$

a The $\mathrm{HR}$ of $\mathrm{HbA}_{1 \mathrm{c}}-\mathrm{MEAN}$ was estimated also for increases of 1-SD in the model with the highest $\mathrm{HR}$ of the glycemic variability parameter, whichever it was

inferences, can be made, but only speculated. Moreover, as with any cohort study, residual confounding due to unmeasured or unknown factors can not be ruled out. Second, it enrolled mainly middle-aged to elderly individuals with long-standing type 2 diabetes and with a high prevalence of chronic complications followed-up in a tertiary-care university hospital. Hence, our results might not be generalized to younger individuals with 
Table 5 Results of Cox survival analyses for the excess risks associated with 12- and 24-month glycemic variability parameters, divided into tertiles, for the occurrence of future macrovascular complications and mortality

\begin{tabular}{|c|c|c|c|c|c|c|c|}
\hline \multirow[t]{2}{*}{ Outcome } & \multirow{2}{*}{$\begin{array}{l}\text { Glycemic } \\
\text { parameter }\end{array}$} & \multicolumn{3}{|c|}{ 12-month glycemic variability } & \multicolumn{3}{|c|}{ 24-month glycemic variability } \\
\hline & & $\begin{array}{l}\text { Model } 1 \\
\text { HR }(95 \% \mathrm{Cl})\end{array}$ & $\begin{array}{l}\text { Model } 2 \\
\text { HR }(95 \% \text { Cl) }\end{array}$ & $\begin{array}{l}\text { Model } 3 \\
\text { HR }(95 \% \text { Cl) }\end{array}$ & $\begin{array}{l}\text { Model } 1 \\
\text { HR }(95 \% \text { Cl) }\end{array}$ & $\begin{array}{l}\text { Model } 2 \\
\text { HR }(95 \% \mathrm{Cl})\end{array}$ & $\begin{array}{l}\text { Model } 3 \\
\text { HR }(95 \% \mathrm{Cl})\end{array}$ \\
\hline \multirow{4}{*}{$\begin{array}{l}\text { Total CV events } \\
n=128\end{array}$} & FG-SD & $2.85(1.79-4.54)^{*}$ & $2.08(1.22-3.54)^{+}$ & $1.71(0.94-3.10)$ & $2.39(1.52-3.77)^{*}$ & $1.60(0.95-2.72)$ & $1.22(0.68-2.21)$ \\
\hline & FG-VC & $2.35(1.49-3.68)^{*}$ & $1.82(1.10-3.01)^{\ddagger}$ & $1.62(0.97-2.71)$ & $2.54(1.62-3.99)^{*}$ & $1.97(1.18-3.31)^{\ddagger}$ & $1.76(1.04-3.00)^{\ddagger}$ \\
\hline & $\mathrm{HbA}_{1 c}-\mathrm{SD}$ & $1.93(1.26-2.95)^{\dagger}$ & $1.44(0.91-2.28)$ & $1.04(0.59-1.82)$ & $1.87(1.22-2.87)^{\dagger}$ & $1.43(0.90-2.25)$ & $1.03(0.59-1.79)$ \\
\hline & $\mathrm{HbA}_{1 \mathrm{c}}-\mathrm{VC}$ & $1.76(1.15-2.71)^{\ddagger}$ & $1.47(0.94-2.29)$ & $1.18(0.72-1.92)$ & $2.01(1.31-3.11)^{\dagger}$ & $1.69(1.07-2.66)^{\ddagger}$ & $1.38(0.84-2.26)$ \\
\hline \multirow{4}{*}{$\begin{array}{l}\text { Major CV events } \\
n=96\end{array}$} & FG-SD & $2.74(1.63-4.61)^{*}$ & $1.83(1.01-3.32)^{\ddagger}$ & $1.37(0.70-2.68)$ & $2.64(1.56-4.48)^{*}$ & $1.64(0.89-3.03)$ & $1.24(0.63-2.44)$ \\
\hline & FG-VC & $1.92(1.16-3.19)^{\ddagger}$ & $1.42(0.81-2.49)$ & $1.31(0.73-2.33)$ & $2.37(1.42-3.97)^{\dagger}$ & $1.69(0.94-3.05)$ & $1.54(0.85-2.80)$ \\
\hline & $\mathrm{HbA}_{1 \mathrm{c}}-\mathrm{SD}$ & $2.47(1.51-4.05)^{*}$ & $1.73(1.03-2.93)^{\ddagger}$ & $1.39(0.75-2.57)$ & $2.42(1.47-3.99)^{\dagger}$ & $1.67(0.98-2.86)$ & $1.30(0.70-2.43)$ \\
\hline & $\mathrm{HbA}_{1 c}-\mathrm{VC}$ & $2.17(1.34-3.51)^{\dagger}$ & $1.71(1.04-2.82)^{\ddagger}$ & $1.43(0.83-2.44)$ & $2.33(1.43-3.79)^{\dagger}$ & $1.78(1.07-2.97)^{\ddagger}$ & $1.49(0.86-2.57)$ \\
\hline \multirow{4}{*}{$\begin{array}{l}\text { CV mortality } \\
\mathrm{n}=67\end{array}$} & FG-SD & $3.26(1.73-6.13)^{*}$ & $2.67(1.28-5.59)^{\dagger}$ & $1.85(0.81-4.24)$ & $3.27(1.72-6.22)^{*}$ & $2.50(1.17-5.33)^{\ddagger}$ & $1.72(0.75-3.96)$ \\
\hline & FG-VC & $2.26(1.20-4.24)^{\ddagger}$ & $1.92(0.94-3.94)$ & $1.60(0.76-3.37)$ & $3.23(1.68-6.21)^{*}$ & $2.82(1.34-5.95)^{\dagger}$ & $2.41(1.13-5.17)^{\ddagger}$ \\
\hline & $\mathrm{HbA}_{1 c}-\mathrm{SD}$ & $3.18(1.77-5.71)^{*}$ & $2.60(1.38-4.91)^{\dagger}$ & $2.07(0.97-4.40)$ & $3.02(1.65-5.51)^{*}$ & $2.40(1.25-4.62)^{\dagger}$ & $1.72(0.80-3.71)$ \\
\hline & $\mathrm{HbA}_{1 c}-\mathrm{VC}$ & $2.74(1.53-4.89)^{\dagger}$ & $2.22(1.22-4.06)^{\dagger}$ & $1.78(0.92-3.44)$ & $2.81(1.57-5.02)^{*}$ & $2.33(1.26-4.32)^{\dagger}$ & $1.81(0.93-3.54)$ \\
\hline \multirow{4}{*}{$\begin{array}{l}\text { All-cause mortality } \\
\mathrm{n}=158\end{array}$} & FG-SD & $3.20(2.14-4.79)^{*}$ & $2.62(1.64-4.17)^{*}$ & $2.60(1.55-4.37)^{*}$ & $2.80(1.86-4.21)^{*}$ & $2.05(1.28-3.27)^{\dagger}$ & $1.82(1.09-3.06)^{\ddagger}$ \\
\hline & FG-VC & $2.45(1.63-3.69)^{*}$ & $2.07(1.31-3.28)^{\dagger}$ & $1.96(1.22-3.14)^{\dagger}$ & $2.71(1.79-4.08)^{*}$ & $2.21(1.39-3.51)^{*}$ & $2.06(1.28-3.31)^{\dagger}$ \\
\hline & $\mathrm{HbA}_{1 c}-\mathrm{SD}$ & $2.15(1.45-3.20)^{*}$ & $1.64(1.07-2.51)^{\ddagger}$ & $1.35(0.80-2.25)$ & $1.95(1.31-2.91)^{*}$ & $1.48(0.96-2.28)$ & $1.10(0.66-1.83)$ \\
\hline & $\mathrm{HbA}_{1 c}-\mathrm{VC}$ & $2.12(1.43-3.15)^{*}$ & $1.72(1.15-2.59)^{\dagger}$ & $1.51(0.97-2.35)$ & $2.19(1.46-3.27)^{*}$ & $1.76(1.16-2.69)^{\dagger}$ & $1.49(0.95-2.34)$ \\
\hline
\end{tabular}

Values are hazard ratios for the highest tertile subgroup in relation to the lowest one and $95 \%$ confidence intervals

Model 1 is adjusted for age, sex and number of $\mathrm{HbA}_{1 \mathrm{c}}$ or $\mathrm{FG}$ measurements

Model 2 is further adjusted for diabetes duration, BMI, smoking status, physical inactivity, arterial hypertension, number of anti-hypertensive drugs in use, ambulatory 24-h SBP, presence of micro- and macrovascular complications at baseline, serum mean HDL- and LDL-cholesterol, and use of insulin, statins and aspirin

Model 3 is further adjusted for mean fasting glycemia and $\mathrm{HbA}_{1 c}$

$H R$ hazard ratio, $C l$ confidence interval, $C V$ cardiovascular, $F G-S D$ fasting glucose standard deviation, $F G-V C$ fasting glucose variation coefficient, $H b A_{1 c}-S D$ glycated hemoglobin standard deviation, $H b A_{1 c}-V C$ glycated hemoglobin variation coefficient

${ }^{*} p<0.001 ;{ }^{\dagger} p<0.01 ;{ }^{\ddagger} p<0.05$

recent onset type 2 diabetes or at primary care management. Third, changes in anti-diabetic medications during follow-up, particularly initiating or increasing insulin dosages, which probably affected glycemic variability during the first 2 years of follow-up, were not taken into account. Forth, peripheral neuropathy assessment was not performed annually during follow-up, as the other outcomes, but on two specific time points (at baseline and after a median of 6 years), which might have affected this endpoint evaluation, although this specific analysis took into account the differential time interval between neuropathy assessments. Finally, we did not adjust for multiple comparisons within each outcome. However, as we have evaluated 4 glycemic variability parameters obtained during 2 time intervals, that is 8 measures for each outcome; if we applied Bonferroni's correction, we would have considered a $\mathrm{p}$ value $<0.006$ as significant. With this more conservative approach, only the predictive capacity of glycemic variability for MACE and microalbuminuria incidence would be lost. On the other hand, this study main strength is its well-documented cohort with standardized care and annual outcomes evaluation over a long follow-up, which permitted the most comprehensive analysis of the associations between long-term glycemic variability parameters and risks of separate micro- and macrovascular complications and of mortality in patients with type 2 diabetes. 
Table 6 Results of multivariable analyses for the excess risks associated with 12- and 24-month glycemic variability parameters, divided into tertiles, for the occurrence of future diabetic microvascular complications

\begin{tabular}{|c|c|c|c|c|c|c|c|}
\hline \multirow[t]{2}{*}{ Outcome } & \multirow{2}{*}{$\begin{array}{l}\text { Glycemic } \\
\text { parameter }\end{array}$} & \multicolumn{3}{|c|}{ 12-month glycemic variability } & \multicolumn{3}{|c|}{ 24-month glycemic variability } \\
\hline & & $\begin{array}{l}\text { Model } 1 \\
\text { HR }(95 \% \mathrm{Cl})\end{array}$ & $\begin{array}{l}\text { Model } 2 \\
\text { HR (95\% Cl) }\end{array}$ & $\begin{array}{l}\text { Model } 3 \\
\text { HR }(95 \% \mathrm{Cl})\end{array}$ & $\begin{array}{l}\text { Model } 1 \\
\text { HR (95\% Cl) }\end{array}$ & $\begin{array}{l}\text { Model } 2 \\
\text { HR }(95 \% \mathrm{Cl})\end{array}$ & $\begin{array}{l}\text { Model } 3 \\
\text { HR }(95 \% \mathrm{Cl})\end{array}$ \\
\hline \multirow{4}{*}{$\begin{array}{l}\text { Retinopathy } \\
\quad \text { (incident or } \\
\text { worsening) } \\
n=152\end{array}$} & FG-SD & $2.78(1.84-4.21)^{*}$ & $1.66(1.04-2.65)^{\ddagger}$ & $1.17(0.69-1.98)$ & $3.24(2.14-4.92)^{*}$ & $1.89(1.18-3.02)^{\dagger}$ & $1.36(0.79-2.33)$ \\
\hline & FG-VC & $2.47(1.64-3.71)^{*}$ & $1.50(0.96-2.33)$ & $1.31(0.82-2.09)$ & $2.47(1.64-3.70)^{*}$ & $1.45(0.93-2.25)$ & $1.23(0.77-1.96)$ \\
\hline & $\mathrm{HbA}_{1 c}-\mathrm{SD}$ & $2.59(1.70-3.94)^{*}$ & $1.68(1.07-2.64)^{\ddagger}$ & $1.13(0.66-1.95)$ & $3.02(1.96-4.65)^{*}$ & $2.01(1.26-3.19)^{\dagger}$ & $1.44(0.84-2.46)$ \\
\hline & $\mathrm{HbA}_{1 c}-\mathrm{VC}$ & $2.03(1.34-3.06)^{\dagger}$ & $1.50(0.98-2.31)$ & $1.16(0.74-1.84)$ & $2.47(1.62-3.76)^{*}$ & $1.82(1.17-2.84)^{\dagger}$ & $1.41(0.88-2.26)$ \\
\hline \multirow{4}{*}{$\begin{array}{l}\text { Composite renal } \\
\text { outcome } \\
n=183\end{array}$} & FG-SD & $1.87(1.29-2.71)^{\dagger}$ & $1.52(1.00-2.31)$ & $1.32(0.82-2.13)$ & $2.18(1.50-3.17)^{*}$ & $1.88(1.23-2.87)^{\dagger}$ & $1.66(1.03-2.67)^{\ddagger}$ \\
\hline & FG-VC & $1.69(1.17-2.45)^{\dagger}$ & $1.49(0.99-2.22)$ & $1.39(0.91-2.12)$ & $1.84(1.28-2.66)^{\dagger}$ & $1.66(1.12-2.51)^{\ddagger}$ & $1.60(1.05-1.43)^{\ddagger}$ \\
\hline & $\mathrm{HbA}_{1 \mathrm{c}}-\mathrm{SD}$ & $1.52(1.06-2.17)^{\ddagger}$ & $1.23(0.84-1.81)$ & $1.00(0.62-1.59)$ & $1.68(1.18-2.39)^{\dagger}$ & $1.38(0.94-2.02)$ & $1.14(0.72-1.81)$ \\
\hline & $\mathrm{HbA}_{1 c}-\mathrm{VC}$ & $1.42(1.00-2.02)^{\ddagger}$ & $1.22(0.85-1.76)$ & $1.06(0.72-1.58)$ & $1.56(1.10-2.21)^{\ddagger}$ & $1.35(0.93-1.94)$ & $1.16(0.79-1.72)$ \\
\hline \multirow{4}{*}{$\begin{array}{l}\text { Microalbuminuria } \\
\quad \text { (incident) } \\
\mathrm{n}=89\end{array}$} & FG-SD & $1.66(0.98-2.81)$ & $1.67(0.93-2.98)$ & $1.60(0.81-3.13)$ & $1.70(1.00-2.90)^{\ddagger}$ & $1.78(0.98-3.22)$ & $1.64(0.81-3.33)$ \\
\hline & FG-VC & $1.32(0.78-2.25)$ & $1.37(0.77-2.42)$ & $1.31(0.73-2.37)$ & $1.63(0.96-2.78)$ & $1.85(1.03-3.30)^{\ddagger}$ & $1.80(0.98-3.30)$ \\
\hline & $\mathrm{HbA}_{1 c}-\mathrm{SD}$ & $1.10(0.66-1.83)$ & $1.04(0.60-1.81)$ & $0.80(0.40-1.60)$ & $1.31(0.79-2.18)$ & $1.28(0.74-2.22)$ & $1.06(0.54-2.11)$ \\
\hline & $\mathrm{HbA}_{1 c}-\mathrm{VC}$ & $1.09(0.65-1.81)$ & $1.05(0.62-1.79)$ & $0.90(0.50-1.63)$ & $1.32(0.79-2.19)$ & $1.27(0.74-2.19)$ & $1.09(0.59-2.01)$ \\
\hline \multirow{4}{*}{$\begin{array}{l}\text { Renal failure } \\
n=91\end{array}$} & FG-SD & $2.70(1.54-4.72)^{*}$ & $1.70(0.90-3.20)$ & $1.36(0.67-2.77)$ & $3.63(2.05-6.45)^{*}$ & $2.14(1.13-4.07)^{\ddagger}$ & $1.75(0.86-3.54)$ \\
\hline & FG-VC & $2.56(1.46-4.50)^{*}$ & $1.86(1.01-3.44)^{\ddagger}$ & $1.63(0.86-3.08)$ & $2.67(1.56-4.59)^{*}$ & $1.71(0.95-3.09)$ & $1.57(0.85-2.88)$ \\
\hline & $\mathrm{HbA}_{1 c}-\mathrm{SD}$ & $3.40(1.95-5.92)^{*}$ & $2.34(1.29-4.24)^{\dagger}$ & $2.12(1.05-4.27)^{\ddagger}$ & $3.12(1.82-5.37)^{*}$ & $2.13(1.19-3.81)^{\ddagger}$ & $1.78(0.91-3.49)$ \\
\hline & $\mathrm{HbA}_{1 c}-\mathrm{VC}$ & $2.43(1.43-4.11)^{*}$ & $1.91(1.10-3.30)^{\ddagger}$ & $1.66(0.92-2.98)$ & $2.38(1.42-4.01)^{*}$ & $1.78(1.03-3.08)^{\ddagger}$ & $1.50(0.84-2.67)$ \\
\hline \multirow{4}{*}{$\begin{array}{l}\text { Peripheral neu- } \\
\text { ropathy (incident } \\
\text { or worsening) } \\
n=96\end{array}$} & FG-SD & $2.50(1.35-4.62)^{\dagger}$ & $1.45(0.70-3.00)$ & $1.05(0.46-2.41)$ & $2.82(1.54-5.13)^{*}$ & $1.73(0.85-3.50)$ & $1.35(0.60-3.03)$ \\
\hline & FG-VC & $3.45(1.77-6.72)^{*}$ & $2.52(1.17-5.39)^{\ddagger}$ & $2.02(0.91-4.45)$ & $3.60(1.91-6.79)^{*}$ & $2.82(1.36-5.87)^{\dagger}$ & $2.33(1.09-4.97)^{\ddagger}$ \\
\hline & $\mathrm{HbA}_{1 c}-\mathrm{SD}$ & $2.07(1.16-3.77)^{\ddagger}$ & $1.35(0.69-2.68)$ & $0.76(0.33-1.75)$ & $2.14(1.18-3.90)^{\ddagger}$ & $1.39(0.70-2.77)$ & $0.79(0.34-1.79)$ \\
\hline & $\mathrm{HbA}_{1 c}-\mathrm{VC}$ & $1.82(1.00-3.34)^{\ddagger}$ & $1.34(0.69-2.62)$ & $0.97(0.47-2.01)$ & $2.38(1.29-4.39)^{\dagger}$ & $1.63(0.83-3.22)$ & $1.20(0.57-2.50)$ \\
\hline \multirow{4}{*}{$\begin{array}{l}\text { Peripheral neurop- } \\
\text { athy (incident) } \\
n=42\end{array}$} & FG-SD & $2.04(0.90-4.62)$ & $1.46(0.54-3.95)$ & $0.99(0.31-3.16)$ & $1.78(0.80-3.97)$ & $1.15(0.44-2.99)$ & $0.73(0.24-2.25)$ \\
\hline & FG-VC & $2.56(1.07-6.13)^{\ddagger}$ & $2.13(0.78-5.81)$ & $1.80(0.64-5.07)$ & $2.46(1.05-5.78)^{\ddagger}$ & $2.18(0.83-5.72)$ & $1.88(0.69-5.13)$ \\
\hline & $\mathrm{HbA}_{1 c}-\mathrm{SD}$ & $2.17(0.98-4.79)$ & $1.55(0.62-3.85)$ & $1.07(0.35-3.33)$ & $2.21(1.00-4.89)^{\ddagger}$ & $1.59(0.64-3.94)$ & $1.15(0.37-3.52)$ \\
\hline & $\mathrm{HbA}_{1 c}-\mathrm{VC}$ & $2.78(1.16-6.67)^{\ddagger}$ & $2.14(0.82-5.58)$ & $1.73(0.61-4.94)$ & $3.06(1.28-7.23)^{\ddagger}$ & $2.22(0.86-5.75)$ & $1.85(0.66-5.18)$ \\
\hline
\end{tabular}

Values are hazard ratios and 95\% confidence intervals, estimated by Cox analyses, for the highest tertile subgroup in relation to the lowest one; except for peripheral neuropathy outcomes that are odds ratios and $95 \%$ confidence intervals, estimated by logistic regressions

Models 1, 2 and 3 were adjusted for the same covariates as in Table 3, except for peripheral neuropathy that was adjusted for height instead of BMI and further for the time interval between baseline and second neuropathy examination

$H R$ hazard ratio, $C l$ confidence interval, $C V$ cardiovascular, $F G$-SD fasting glucose standard deviation, $F G-V C$ fasting glucose variation coefficient, $H b A_{1 c}-S D$ glycated hemoglobin standard deviation, $H b A_{1 c}-V C$ glycated hemoglobin variation coefficient

${ }^{*} p<0.001{ }^{\dagger} p<0.01{ }^{\ddagger} p<0.05$

\section{Conclusions}

This prospective cohort study with a long follow-up of high cardiovascular risk individuals with type 2 diabetes provides evidence that 24-month visit-to-visit $\mathrm{HbA}_{1 \mathrm{c}}$ and FG variabilities are better risk predictors than mean $\mathrm{HbA}_{1 \mathrm{c}}$ levels for all micro- and macrovascular complications and all-cause mortality outcomes, except for retinopathy development/progression in patients with poorly-controlled diabetes and for peripheral neuropathy progression. Reducing glycemic variability can be achieved [62]; however, whether this reduction would translate into better prognosis in patients with diabetes, it still remains to be demonstrated. A single randomized trial [63] in post-myocardial infarction patients with type 2 diabetes did not demonstrated any benefit for cardiovascular outcomes, although the differences in glycemic variability (mainly postprandial glycemia) between the two groups were small, which precluded the demonstration of any benefit [42]. More randomized trials on this issue are needed before we may recommend moving from mean $\mathrm{HbA}_{1 \mathrm{c}}$ levels to glycemic variability as the main parameter of glycemic control monitoring. However, until then, glycemic variability parameter shall at least be measured as an additional parameter to improve risk stratification in patients with type 2 diabetes. 


\section{Abbreviations}

ABPM: ambulatory blood pressure monitoring; BMl: body mass index; BP: blood pressure; Cl: confidence interval; CVE: cardiovascular events; DBP: diastolic blood pressure; eGFR: estimated glomerular filtration rate; FG: fasting glycaemia; $\mathrm{HbA}_{1 c}$ : glycated hemoglobin; HR: hazard ratio; MACE: major adverse cardiovascular events; OR: odds ratio; SBP: systolic blood pressure; SD: standard deviation; VC: variation coefficient.

\section{Authors' contributions}

CRLC, NCL and GFS conceived and designed the study. All authors followedup the patients and obtained the data. CRLC drafted the manuscript. GFS analyzed the data and is the guarantor. All authors helped interpret the results and reviewed the manuscript. GFS had full access to all of the data and takes responsibility for the integrity of the data and the accuracy of data analysis. All authors read and approved the final manuscript.

\section{Author details}

${ }^{1}$ Department of Internal Medicine, University Hospital Clementino Fraga Filho, School of Medicine, Universidade Federal do Rio de Janeiro, Rua Croton, 72, Jacarepagua, Rio de Janeiro, RJ CEP: 22750-240, Brazil. ${ }^{2}$ Department of Occupational Therapy, University Hospital Clementino Fraga Filho, School of Medicine, Universidade Federal do Rio de Janeiro, Rio de Janeiro, Brazil.

\section{Acknowledgements}

Not applicable.

\section{Competing interests}

The authors declare that they have no competing interests.

\section{Availability of data and materials}

The Rio de Janeiro Type 2 Diabetes Cohort Study is an on-going study, and its dataset is not publicly available due to individual privacy of the participants. However, it may be available from the corresponding author on reasonable request.

\section{Consent for publication}

Not applicable.

\section{Ethics approval and consent to participate}

The study was approved by the Research Ethics Committee of School of Medicine and University Hospital, Federal University of Rio de Janeiro (Number 124/2004), and all participants gave written informed consent.

\section{Funding}

This study was supported by grants from the Conselho Nacional de Desenvolvimento Científico e Tecnológico (CNPq, Brazil) and from the Fundação Carlos Chagas Filho de Amparo a Pesquisa do Estado do Rio de Janeiro (FAPERJ, Brazil). The sponsors have no role in study design, data collection and analysis, results interpretation or in preparation, review and approval of the manuscript.

\section{Publisher's Note}

Springer Nature remains neutral with regard to jurisdictional claims in published maps and institutional affiliations.

Received: 11 January 2018 Accepted: 16 February 2018 Published online: 24 February 2018

\section{References}

1. Zimmet PZ, Magliano DJ, Herman WH, Shaw JE. Diabetes: a 21st century challenge. Lancet Diabetes Endocrinol. 2014;2:56-64.

2. Ward A, Alvarez P, Vo L, Martin S. Direct medical costs of complications of diabetes in the United States: estimates for event-year and annual state costs (USD 2012). J Med Econ. 2014;17:176-83.

3. American Diabetes Association. Standards of medical care in diabetes-2017. Diabetes Care. 2017;40(Suppl. 1):S48-56.

4. Zhang Y, Hu G, Yuan Z, Chen L. Glycosylated hemoglobin in relationship to cardiovascular outcomes and death in patients with type 2 diabetes: a systematic review and meta-analysis. PLoS ONE. 2012;7:e42551.

5. Holman RR, Paul SK, Bethel MA, Matthews DR, Neil HA. 10-year followup of intensive glucose control in type 2 diabetes. N Engl J Med. 2008:359:1577-89.

6. Hayward RA, Reaven PD, Wiitala WL, Bahn GD, Reda DJ, Ge L, VADT Investigators, et al. Follow-up of glycemic control and cardiovascular outcomes in type 2 diabetes. N Engl J Med. 2015;372:2197-206.

7. Action to Control Cardiovascular Risk in Diabetes Study Group, Gerstein HC, Miller ME, Byington RP, Goff DC Jr, Bigger JT, Buse JB, et al. Effects of intensive glucose lowering in type 2 diabetes. N Engl J Med. 2008;358:2545-59.

8. Zoungas S, Chalmers J, Neal B, Billot L, Li Q, Hirakawa Y, ADVANCE-ON Collaborative Group, et al. Follow-up of blood-pressure lowering and glucose control in type 2 diabetes. N Engl J Med. 2014;371:1392-406.

9. The Diabetes Control and Complications Trial Research Group. The relationship of glycemic exposure $(\mathrm{HbA} 1 \mathrm{c})$ to the risk of development and progression of retinopathy in the diabetes control and complications trial. Diabetes. 1995;44:968-83.

10. Kilpatrick ES, Rigby AS, Atkin SL. A1C variability and the risk of microvascular complications in type 1 diabetes: data from the Diabetes Control and Complications Trial. Diabetes Care. 2008;31:2198-202.

11. Brownlee M, Hirsch IB. Glycemic variability: a hemoglobin A1c-independent risk factor for diabetic complications. JAMA. 2006;295:1707-8.

12. Gorst C, Kwok CS, Aslam S, Buchan I, Kontopantelis E, Myint PK, et al. Long-term glycemic variability and risk of adverse outcomes: a systematic review and meta-analysis. Diabetes Care. 2015;38:2354-69.

13. Gimeno-Orna JA, Castro-Alonso FJ, Boned-Juliani B, Lou-Arnal LM. Fasting plasma glucose variability as a risk factor of retinopathy in type 2 diabetic patients. J Diabetes Complicat. 2003;17:78-81.

14. Zoppini G, Verlato G, Targher G, Casati S, Gusson E, Biasi V, et al. Is fasting glucose variability a risk factor for retinopathy in people with type 2 diabetes? Nutr Metab Cardiovasc Dis. 2009;19:334-9.

15. Takao T, Ide T, Yanagisawa H, Kikuchi M, Kawazu S, Matsuyama Y. The effect of fasting plasma glucose variability on the risk of retinopathy in type 2 diabetic patients: retrospective long-term follow-up. Diabetes Res Clin Pract. 2010;89:296-302

16. Penno G, Solini A, Bonora E, Fondelli C, Orsi E, Zerbini G, Renal Insufficiency And Cardiovascular Events Study Group, et al. HbA1c variability as an independent correlate of nephropathy, but not retinopathy, in patients with type 2 diabetes: the renal insufficiency and cardiovascular events (RIACE) Italian multicenter study. Diabetes Care. 2013;36:2301-10.

17. Foo V, Quah J, Cheung G, Tan NC, Ma Zar KL, Chan CM, et al. HbA1C, systolic blood pressure variability and diabetic retinopathy in Asian type 2 diabetics. J Diabetes. 2017;9:200-7.

18. Takao T, Suka M, Yanagisawa H, Matsuyama Y, Iwamoto Y. Predictive ability of visit-to-visit variability in $\mathrm{HbA1c}$ and systolic blood pressure for the development of microalbuminuria and retinopathy in people with type 2 diabetes. Diabetes Res Clin Pract. 2017;128:15-23.

19. Cummings DM, Larsen LC, Doherty L, Lea CS, Holbert D. Glycemic control patterns and kidney disease progression among primary care patients with diabetes mellitus. J Am Board Fam Med. 2011;24:391-8.

20. Hsu CC, Chang HY, Huang MC, Hwang SJ, Yang YC, Lee YS, et al. $\mathrm{HbA1c}$ variability is associated with microalbuminuria development in type 2 diabetes: a 7-year prospective cohort study. Diabetologia. 2012;55:3163-72

21. Sugawara A, Kawai K, Motohashi S, Saito K, Kodama S, Yachi Y, et al. $\mathrm{HbA}(1 \mathrm{c})$ variability and the development of microalbuminuria in type 2 diabetes: Tsukuba Kawai Diabetes Registry 2. Diabetologia. 2012:55:2128-31.

22. Rodríguez-Segade S, Rodríguez J, García López JM, Casanueva FF, Camiña F. Intrapersonal $\mathrm{HbA}(1 \mathrm{c})$ variability and the risk of progression of nephropathy in patients with type 2 diabetes. Diabet Med. 2012;29:1562-6.

23. Luk AO, Ma RC, Lau ES, Yang X, Lau WW, Yu LW, et al. Risk association of $\mathrm{HbA1}$ c variability with chronic kidney disease and cardiovascular disease in type 2 diabetes: prospective analysis of the Hong Kong Diabetes Registry. Diabetes Metab Res Rev. 2013;29:384-90.

24. Lin CC, Chen CC, Chen FN, Li Cl, Liu CS, Lin WY, et al. Risks of diabetic nephropathy with variation in hemoglobin A1c and fasting plasma glucose. Am J Med. 2013;126:1017.e1-10. 
25. Dorajoo SR, Ng JSL, Goh JHF, Lim SC, Yap CW, Chan A, et al. HbA1C variability in type 2 diabetes is associated with the occurrence of new-onset albuminuria within three years. Diabetes Res Clin Pract. 2017;128:32-9.

26. Low S, Lim SC, Yeoh LY, Liu YL, Liu JJ, Fun S, et al. Effect of long-term glycemic variability on estimated glomerular filtration rate decline among patients with type 2 diabetes mellitus: insights from the Diabetic Nephropathy Cohort in Singapore. J Diabetes. 2017;9:908-19.

27. Jun JE, Lee SE, Lee YB, Ahn JY, Kim G, Jin SM, et al. Glycated albumin and its variability as an indicator of cardiovascular autonomic neuropathy development in type 2 diabetic patients. Cardiovasc Diabetol. 2017:16:127.

28. Bouchi R, Babazono T, Mugishima M, Yoshida N, Nyumura I, Toya K, et al. Fluctuations in $\mathrm{HbA} 1 \mathrm{c}$ are associated with a higher incidence of cardiovascular disease in Japanese patients with type 2 diabetes. J Diabetes Investig. 2012;3:148-55

29. Hirakawa Y, Arima H, Zoungas S, Ninomiya T, Cooper M, Hamet P, et al. Impact of visit-to-visit glycemic variability on the risks of macrovascular and microvascular events and all-cause mortality in type 2 diabetes: the ADVANCE trial. Diabetes Care. 2014;37:2359-65.

30. Takao T, Matsuyama Y, Suka M, Yanagisawa H, Iwamoto Y. The combined effect of visit-to-visit variability in $\mathrm{HbA} 1 \mathrm{c}$ and systolic blood pressure on the incidence of cardiovascular events in patients with type 2 diabetes. BMJ Open Diabetes Res Care. 2015;3:e000129.

31. Bonke FC, Donnachie E, Schneider A, Mehring M. Association of the average rate of change in $\mathrm{HbA} 1 \mathrm{c}$ with severe adverse events: a longitudinal evaluation of audit data from the Bavarian Disease Management Program for patients with type 2 diabetes mellitus. Diabetologia. 2016;59:286-93.

32. Prentice JC, Pizer SD, Conlin PR. Identifying the independent effect of $\mathrm{HbA1c}$ variability on adverse health outcomes in patients with type 2 diabetes. Diabet Med. 2016;33:1640-8.

33. Wan EY, Fung CS, Fong DY, Lam CL. Association of variability in hemoglobin A1c with cardiovascular diseases and mortality in Chinese patients with type 2 diabetes mellitus - a retrospective population-based cohort study. J Diabetes Complicat. 2016;30:1240-7.

34. Laiteerapong N, Karter AJ, Moffet HH, Cooper JM, Gibbons RD, Liu JY, et al. Ten-year hemoglobin A1c trajectories and outcomes in type 2 diabetes mellitus: the Diabetes \& Aging Study. J Diabetes Complicat. 2017:31:94-100

35. Lee MY, Hsiao PJ, Huang YT, Huang JC, Hsu WH, Chen SC, et al. Greater $\mathrm{HbA1c}$ variability is associated with increased cardiovascular events in type 2 diabetes patients with preserved renal function, but not in moderate to advanced chronic kidney disease. PLoS ONE. 2017:12:e0178319.

36. Zinman B, Marso SP, Poulter NR, Emerson SS, Pieber TR, Pratley RE, DEVOTE Study Group, et al. Day-to-day fasting glycaemic variability in DEVOTE: associations with severe hypoglycaemia and cardiovascular outcomes (DEVOTE 2). Diabetologia. 2018;61:48-57.

37. Muggeo M, Zoppini G, Bonora E, Brun E, Bonadonna RC, Moghetti P, et al. Fasting plasma glucose variability predicts 10-year survival of type 2 diabetic patients: the Verona Diabetes Study. Diabetes Care. 2000;23:45-50.

38. Lin CC, Li Cl, Yang SY, Liu CS, Chen CC, Fuh MM, et al. Variation of fasting plasma glucose: a predictor of mortality in patients with type 2 diabetes. Am J Med. 2012;125:416.e9-18.

39. Ma WY, Li HY, Pei D, Hsia TL, Lu KC, Tsai LY, et al. Variability in hemoglobin A1c predicts all-cause mortality in patients with type 2 diabetes. J Diabetes Complicat. 2012;26:296-300.

40. Takao T, Matsuyama Y, Yanagisawa H, Kikuchi M, Kawazu S. Association between $\mathrm{HbA} 1 \mathrm{c}$ variability and mortality in patients with type 2 diabetes. J Diabetes Complicat. 2014;28:494-9.

41. Skriver MV, Sandbæk A, Kristensen JK, Støvring H. Relationship of HbA1C variability, absolute changes in $\mathrm{HbA} 1 \mathrm{c}$, and all-cause mortality in type 2 diabetes: a Danish population-based prospective observational study. BMJ Open Diabetes Res Care. 2015;3:e000060.

42. Hirsch IB. Glycemic variability and diabetes complications: does it matter? Of course it does! Diabetes Care. 2015;38:1610-4

43. Bergenstal RM. Glycemic variability and diabetes complications: does it matter? Simply put, there are better glycemic markers! Diabetes Care. 2015;38:1615-21.

44. Cardoso CR, Leite NC, Ferreira MT, Salles GF. Prognostic importance of baseline and serial glycated hemoglobin levels in high-risk patients with type 2 diabetes: The Rio de Janeiro Type 2 Diabetes Cohort Study. Acta Diabetol. 2015:52:21-9.
45. Cardoso CR, Moran CB, Marinho FS, Ferreira MT, Salles GF, Increased aortic stiffness predicts future development and progression of peripheral neuropathy in patients with type 2 diabetes: The Rio de Janeiro Type 2 Diabetes Cohort Study. Diabetologia. 2015;58:2161-8.

46. Cardoso CRL, Leite NC, Dib E, Salles GF. Predictors of development and progression of retinopathy in patients with type 2 diabetes: importance of blood pressure parameters. Sci Rep. 2017;7:4867.

47. Cardoso CRL, Leite NC, Salles GC, Ferreira MT, Salles GF. Aortic stiffness and ambulatory blood pressure as predictors of diabetic kidney disease: a competing risks analysis from The Rio de Janeiro Type 2 Diabetes Cohort Study. Diabetologia. 2018;61:455-65.

48. Niskanen L, Virkamäki A, Hansen JB, Saukkonen T. Fasting plasma glucose variability as a marker of nocturnal hypoglycemia in diabetes: evidence from the PREDICTIVE study. Diabetes Res Clin Pract. 2009:86:e15-8.

49. Pieber TR, Marso SP, McGuire DK, Zinman B, Poulter NR, Emerson SS, DEVOTE Study Group, et al. DEVOTE 3: temporal relationships between severe hypoglycaemia, cardiovascular outcomes and mortality. Diabetologia. 2018;61:58-65.

50. Rutter MK. Devoting attention to glucose variability and hypoglycaemia in type 2 diabetes. Diabetologia. 2018:61:43-7.

51. Ouchi S, Shimada K, Miyazaki T, Takahashi S, Sugita Y, Shimizu M, et al. Low 1,5-anhydroglucitol levels are associated with long-term cardiac mortality in acute coronary syndrome patients with hemoglobin A1c levels less than 7.0. Cardiovasc Diabetol. 2017;16:151.

52. Takahashi S, Shimada K, Miyauchi K, Miyazaki T, Sai E, Ogita M, et al. Low and exacerbated levels of 1,5-anhydroglucitol are associated with cardiovascular events in patients after first-time elective percutaneous coronary intervention. Cardiovasc Diabetol. 2016;15:145.

53. Monnier L, Mas E, Ginet C, Michel F, Villon L, Cristol JP, et al. Activation of oxidative stress by acute glucose fluctuations compared with sustained chronic hyperglycemia in patients with type 2 diabetes. JAMA. 2006;295:1681-7

54. Ceriello A, Esposito K, Piconi L, Ihnat MA, Thorpe JE, Testa R, et al. Oscillating glucose is more deleterious to endothelial function and oxidative stress than mean glucose in normal and type 2 diabetic patients. Diabetes. 2008:57:1349-54.

55. Kuroda M, Shinke T, Otake H, Sugiyama D, Takaya T, Takahashi H, et al. Effects of daily glucose fluctuations on the healing response to everolimus-eluting stent implantation as assessed using continuous glucose monitoring and optical coherence tomography. Cardiovasc Diabetol. 2016;15:79

56. Zhang JW, He LJ, Cao SJ, Yang Q, Yang SW, Zhou YJ. Effect of glycemic variability on short term prognosis in acute myocardial infarction subjects undergoing primary percutaneous coronary interventions. Diabetol Metab Syndr. 2014;6:76.

57. Xia J, Xu J, Hu S, Hao H, Yin C, Xu D. Impact of glycemic variability on the occurrence of periprocedural myocardial infarction and major adverse cardiovascular events (MACE) after coronary intervention in patients with stable angina pectoris at 6 months follow-up. Clin Chim Acta. 2017;471:196-200.

58. Schisano B, Tripathi G, McGee K, McTernan PG, Ceriello A. Glucose oscillations, more than constant high glucose, induce p53 activation and a metabolic memory in human endothelial cells. Diabetologia. 2011;54:1219-26.

59. Keating ST, El-Osta A. Glycemic memories and the epigenetic component of diabetic nephropathy. Curr Diab Rep. 2013;13:574-81.

60. Groop PH, Forsblom C, Thomas MC. Mechanisms of disease: pathwayselective insulin resistance and microvascular complications of diabetes. Nat Clin Pract Endocrinol Metab. 2005;1:100-10.

61. Del Guerra S, Grupillo M, Masini M, Lupi R, Bugliani M, Torri S, et al. Gliclazide protects human islet beta-cells from apoptosis induced by intermittent high glucose. Diabetes Metab Res Rev. 2007;23:234-8.

62. FLAT-SUGAR Trial Investigators. Glucose variability in a 26-week randomized comparison of mealtime treatment with rapid-acting insulin versus GLP-1 agonist in participants with type 2 diabetes at high cardiovascular risk. Diabetes Care. 2016:39:973-81.

63. Siegelaar SE, Kerr L, Jacober SJ, Devries JH. A decrease in glucose variability does not reduce cardiovascular event rates in type 2 diabetic patients after acute myocardial infarction: a reanalysis of the HEART2D study. Diabetes Care. 2011;34:855-7. 